\author{
Marquette University \\ e-Publications@Marquette
}

2008

\title{
A Second-Generation Janus Scorpionate Ligand: Controlling Coordination Modes in Iron(II) Complexes by Steric Modulation
}

\author{
Rosalice M. Silva \\ Marquette University \\ Chengeto Gwengo \\ Marquette University \\ Sergey V. Lindeman \\ Marquette University, sergey.lindeman@marquette.edu \\ Mark D. Smith \\ University of South Carolina \\ Gary J. Long \\ University of Missouri
}

See next page for additional authors

Follow this and additional works at: https://epublications.marquette.edu/chem_fac

Part of the Chemistry Commons

\section{Recommended Citation}

Silva, Rosalice M.; Gwengo, Chengeto; Lindeman, Sergey V.; Smith, Mark D.; Long, Gary J.; Grandjean, Fernande; and Gardinier, James R., "A Second-Generation Janus Scorpionate Ligand: Controlling Coordination Modes in Iron(II) Complexes by Steric Modulation" (2008). Chemistry Faculty Research and Publications. 619.

https://epublications.marquette.edu/chem_fac/619 


\section{Authors}

Rosalice M. Silva, Chengeto Gwengo, Sergey V. Lindeman, Mark D. Smith, Gary J. Long, Fernande Grandjean, and James R. Gardinier 


\section{Marquette University}

\section{e-Publications@Marquette}

\section{Chemistry Faculty Research and Publications/College of Arts and Sciences}

This paper is NOT THE PUBLISHED VERSION; but the author's final, peer-reviewed manuscript. The published version may be accessed by following the link in th citation below.

Inorganic Chemistry, Vol. 47, No. 16 (2008): 7233-7242. DOI. This article is (C) American Chemical Society and permission has been granted for this version to appear in e-Publications@Marquette. American Chemical Society does not grant permission for this article to be further copied/distributed or hosted elsewhere without the express permission from American Chemical Society.

\section{A Second-Generation Janus Scorpionate Ligand: Controlling Coordination Modes in Iron(II) Complexes by Steric Modulation}

Rosalice M. Silva

Department of Chemistry, Marquette University, Milwaukee, WI

Chengeto Gwengo

Department of Chemistry, Marquette University, Milwaukee, WI

Sergey V. Lindeman

Department of Chemistry, Marquette University, Milwaukee, WI

Mark D. Smith

Department of Chemistry and Biochemistry, University of South Carolina, Columbia, SC

Gary J. Long

Department of Chemistry, Missouri University of Science and Technology, University of Missouri, Rolla, Missouri Fernande Grandjean

Department of Physics, B5, University of Liège, B-4000 Sart-Tilman, Belgium

James R. Gardinier

Department of Chemistry, Marquette University, Milwaukee, WI 


\section{Synopsis}

The second-generation Janus scorpionate ligand $\left[\mathrm{HB}\left(\mathrm{mtda}^{\mathrm{Me}}\right)_{3}^{-}\right]$containing methyl-mercaptothiadiazolyl (mtdame) heterocyclic rings and $(N, N, N-)$ and $(S, S, S-)$ binding pockets has been prepared. The effect of methyl substitution versus the unsubstituted first-generation Janus scorpionate $\left[\mathrm{HB}(\mathrm{mtda})_{3}\right]-$ on the coordination chemistry with alkali metals and on the binding preferences and on the ground spin state of iron(II) complexes has been studied structurally and by ${ }^{57} \mathrm{Fe}$ Mossbauer Spectroscopy.

\section{Abstract}
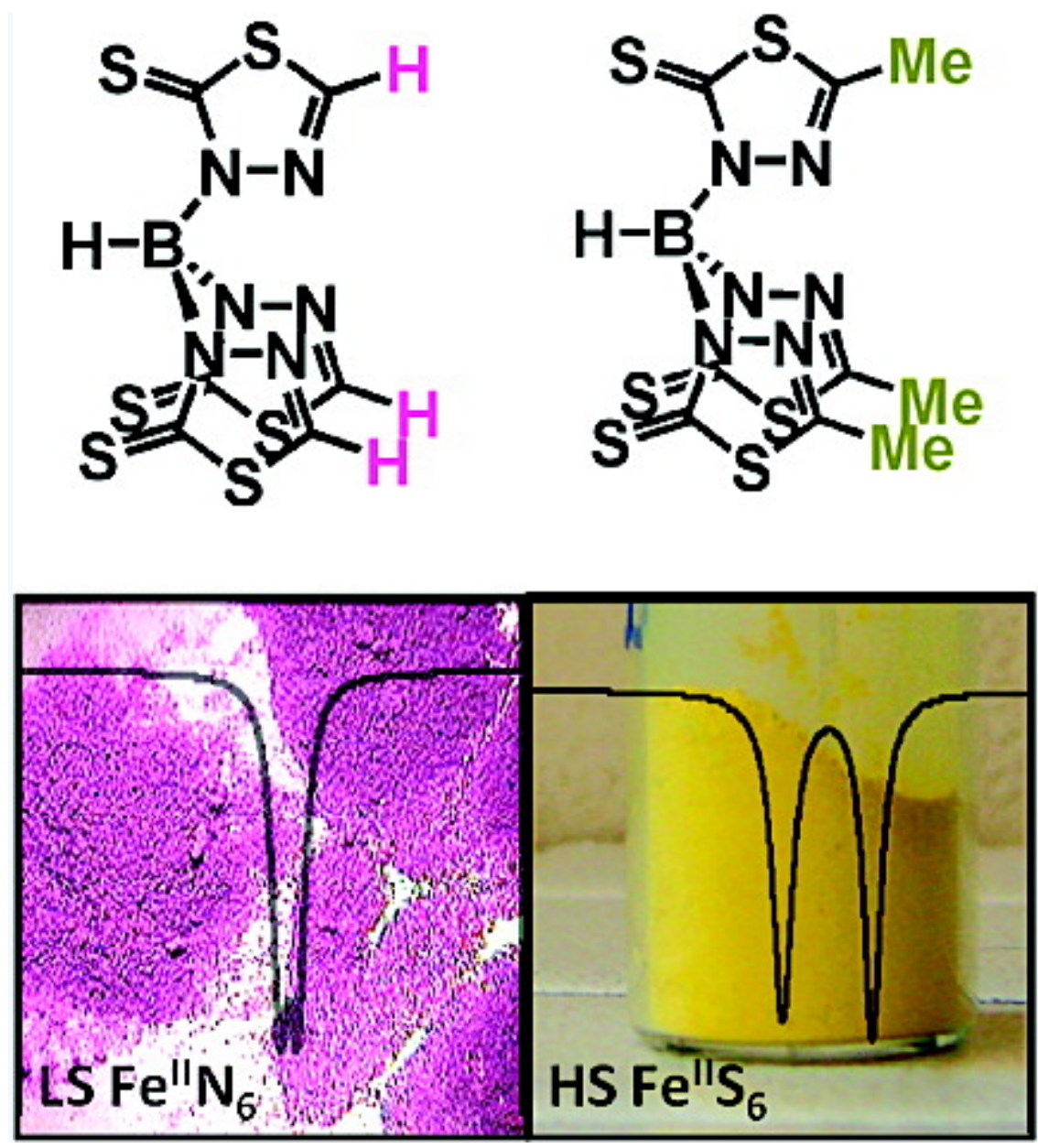

The second-generation Janus scorpionate ligand $\left[\mathrm{HB}\left(\mathrm{mtda}^{\mathrm{Me}}\right)_{3}\right]^{-}\left(\mathrm{mtda}^{\mathrm{Me}}=2\right.$-mercapto-5-methyl-1,3,4thiadiazolyl) with conjoined $(\mathrm{N}, \mathrm{N}, \mathrm{N}-)$ and $(\mathrm{S}, \mathrm{S}, \mathrm{S}-)$ donor faces has been prepared. This second-generation Janus scorpionate ligand $\left[\mathrm{HB}\left(\mathrm{mtda}_{\mathrm{Me}}\right)_{3}\right]^{-}$- differs from the first-generation $\left[\mathrm{HB}(\mathrm{mtda})_{3}\right]^{-}$ligand by the replacement of hydrogens on the heterocyclic rings proximal to the nitrogenous face with methyl groups. This study probed whether steric interactions introduced by such methyl group substitution could modulate the reactivity and coordination preferences of these ambidentate ligands. The crystal structures of a sodium complex $\mathrm{Na}\left[\mathrm{HB}\left(\mathrm{mtda}^{\mathrm{Me}}\right)_{3}\right] \cdot 3(\mathrm{MeOH})$, the potassium complexes $\mathrm{K}\left[\mathrm{HB}(\mathrm{mtda})_{3}\right] \cdot \mathrm{MeOH}$, and $\mathrm{K}_{2}\left[\mathrm{HB}\left(\mathrm{mtda}^{\mathrm{Me}}\right)_{3}\right]_{2} \cdot 3 \mathrm{MeOH}$, and several iron complexes were obtained. The difference between first- and second-generation Janus scorpionate ligands is most obvious from the discrepancy between the properties and structures of the two iron(II) compounds with the formula $\mathrm{Fe}\left[\mathrm{HB}\left(\mathrm{mtda}^{\mathrm{R}}\right)_{3}\right]_{2} \cdot 4 \mathrm{DMF}(\mathrm{R}=\mathrm{H}$ or $\mathrm{Me})$. The complex with the first-generation ligand $(\mathrm{R}=\mathrm{H})$ is pink and diamagnetic. An X-ray structural study revealed two facially coordinated $\mathrm{K}^{3} \mathrm{~N}$-scorpionates 
with no bound solvent molecules. The average Fe-N bond distance of $1.97 \AA$ is indicative of the low-spin $\mathrm{t}_{2 \mathrm{~g}}{ }^{6} \mathrm{e}_{\mathrm{g}}{ }^{{ }_{0}}$ electron configuration. In contrast, the iron(II) complex of the second-generation ligand $(\mathrm{R}=\mathrm{Me})$ is yellow and paramagnetic. This structure shows two trans- $\mathrm{K}^{1} \mathrm{~S}$-scorpionates and four equatorial-bound DMF where the average $\mathrm{Fe}-\mathrm{O}$ and Fe-S distances of 2.12 and $2.51 \AA$, respectively, are indicative of the high-spin $\mathrm{t}_{2 \mathrm{~g}}{ }^{4} \mathrm{e}_{\mathrm{g}}{ }^{{ }^{2}} \mathrm{electron}$ configuration. The discrepancy in binding modes and spin-states of iron(II) is carried over to the solvent-free $\mathrm{Fe}\left[\mathrm{HB}\left(\mathrm{mtda}^{\mathrm{R}}\right)_{3}\right]_{2}(\mathrm{R}=\mathrm{H}, \mathrm{Me})$ complexes, as determined from Mössbauer spectral studies. The Mössbauer spectral parameters for $\mathrm{Fe}\left[\mathrm{HB}(\mathrm{mtda})_{3}\right]_{2}$ are fully consistent with low-spin iron(II) in a Fe $\mathrm{N}_{6}$ environment, whereas those for $\mathrm{Fe}\left[\mathrm{HB}\left(\mathrm{mtda}^{\mathrm{Me}}\right)_{3}\right]_{2}$ are most consistent with high-spin iron(II) in a $\mathrm{FeS}_{6}$ environment. Interestingly, when either complex is dissolved in highly polar solvents (DMF, DMSO, or $\mathrm{H}_{2} \mathrm{O}$ ), the ligand completely dissociates forming $\left[\mathrm{Fe}(\text { solvent })_{6}\right]\left[\mathrm{HB}(\mathrm{mtda})_{3}\right]_{2}(\mathrm{R}=\mathrm{H}, \mathrm{Me})$.

\section{Introduction}

Trofimenko's seminal report on the preparation and reactivity of poly(pyrazolyl)borates instigated countless new areas of research and discovery in the field of coordination chemistry. (1) The simplicity of preparation and structural diversity of the tris(pyrazolyl)borates or so-called scorpionates (Figure 1 ) greatly facilitates systematic chemical investigations and perpetuates their popularity. One important

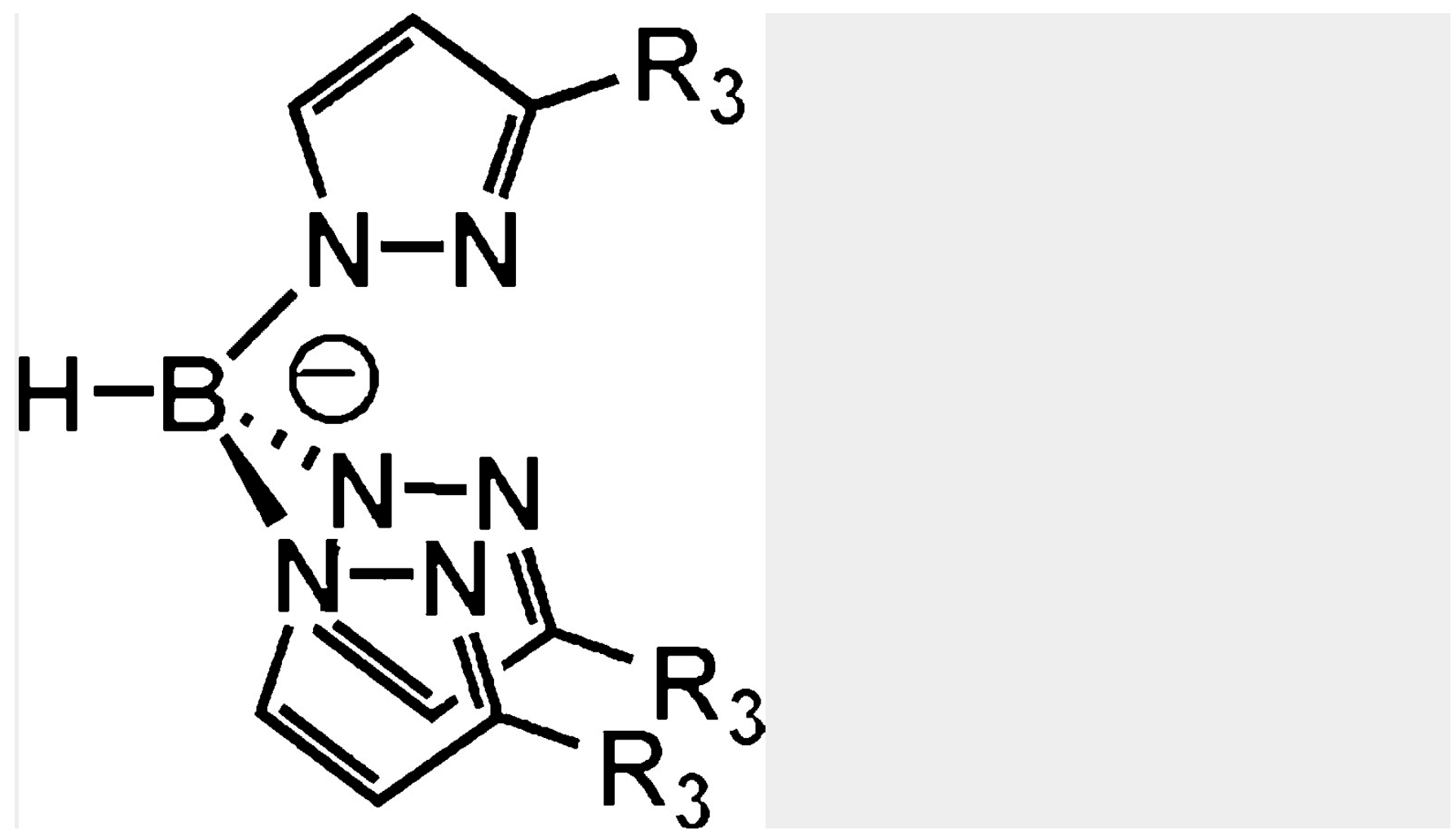

Figure 1. Tris(pyrazolyl)borate ligand with substituent $R$ at the 3-position of the pyrazolyl.

structural modification of the scorpionate manifold occurs by varying the steric demand of substituents at the 3position of the pyrazolyl, nearest to the metal center, as this alteration imparts dramatic changes in ligand reactivity and in the properties of the resulting metal complexes. A distinction can be made between firstgeneration and second-generation scorpionates when the steric demand of the substituents at the 3-position of the pyrazolyl significantly alters the coordination chemistry of the ligand or complex thereof. For instance, in iron chemistry, homoleptic pseudooctahedral iron(II) species $\mathrm{Fe}\left[\mathrm{HB}\left(\mathrm{pz}^{\mathrm{R}}\right)_{3}\right]_{2}$ are obtained from reaction between 
the ligands and an iron(II) dihalide when $\mathrm{R}$ is either hydrogen or methyl.(2) However, when $\mathrm{R}$ is a tert-butyl substituent,(3) heteroleptic pseudotetrahedral complex $\mathrm{Fe}(\mathrm{Cl})\left[\mathrm{HB}\left(\mathrm{pz}^{\mathrm{tBu}}\right)_{3}\right]$ is obtained and the scorpionate ligand in this latter case can therefore be considered second-generation. Interestingly, in the pseudooctahedral $\mathrm{Fe}\left[\mathrm{HB}\left(\mathrm{pz}^{\mathrm{R}}\right)_{3}\right]_{2}$, the parent complex $(\mathrm{R}=\mathrm{H})$ is low spin with $\mathrm{t}_{2 \mathrm{~g}}{ }^{6} \mathrm{eg}_{\mathrm{g}}{ }^{* 0}$ electron configuration at room temperature, whereas, for $\mathrm{R}=\mathrm{Me}$, steric interactions between methyl groups enforce the high spin state $\left(\mathrm{t}_{2 g}{ }^{4} \mathrm{e}_{\mathrm{g}}{ }^{2}\right)$. (4) In both cases, the ligand field strengths are comparable to the electron pairing energy, and spin crossover is observed on changing pressure, temperature, or on light excitation.(5)

More recently, there has been interest in the chemistry of scorpionates that incorporate softer Lewis base donors such as phosphorus (6) or sulfur (7-12) because these donors can be exploited for the preferential binding or stabilization of unusual low-valent, electron-rich, transition-metal centers (or heavier main-group metals). One of the more extensively studied classes of soft scorpionates is the tris(2-mercapto-1-Rimidazolyl)hydroborates, $\left[\mathrm{HB}\left(\mathrm{mim}^{\mathrm{R}}\right)_{3}\right]^{-}$or $\left[\mathrm{Tm}^{\mathrm{R}}\right]^{-}$(Figure 2) introduced by Reglinski, Spicer, and coworkers.(12) The synthetic accessibility and structural diversity again play an important role in the increasing popularity of these ligands. With the $\left[\mathrm{Tm}^{\mathrm{R}}\right]^{-}$ligands, the organic $\mathrm{R}$ substituents are far enough removed from the metal center that steric interactions in fac-coordinated complexes are less pronounced than in the tris(pyrazolyl)borates (even for bulky substituents such as tert-butyls). However, the electronic impact of varying $\mathrm{R}$ groups in the $\mathrm{Tm}^{\mathrm{R}}$ ligands is significant and, most importantly, these ligands often enjoy unexpected binding modes and new reaction chemistry compared to the tris(pyrazolyl)borate counterparts. With iron(II), both pseudo-octahedral Fe $\left[\mathrm{k}^{3}-\mathrm{Tm}^{\mathrm{R}}\right]_{2}{ }^{13}$ and pseudotetrahedral $\mathrm{Fe}(\mathrm{Cl})\left[\mathrm{k}^{3}-\mathrm{Tm}^{\mathrm{R}}\right]^{14}$ can be isolated; for the tris(pyrazolyl)borates the isolation of both $\mathrm{MTp}^{\mathrm{R}}{ }_{2}$ and $\mathrm{MTp}^{\mathrm{R} X}$ is usually not possible for the same $\mathrm{R}$ group. Interestingly, in the case of $\mathrm{Tm}^{\mathrm{tBu}}$, an unusual ligand reaction provided an iron boratrane $\mathrm{Fe}\left[\mathrm{K}^{4} \mathrm{BS}_{3}-\mathrm{Tm}^{\mathrm{tBu}}\right](\mathrm{CO})_{2}$ with a $\mathrm{Fe} \rightarrow \mathrm{B}$ dative bond that can be exploited for further reaction chemistry.(15) 


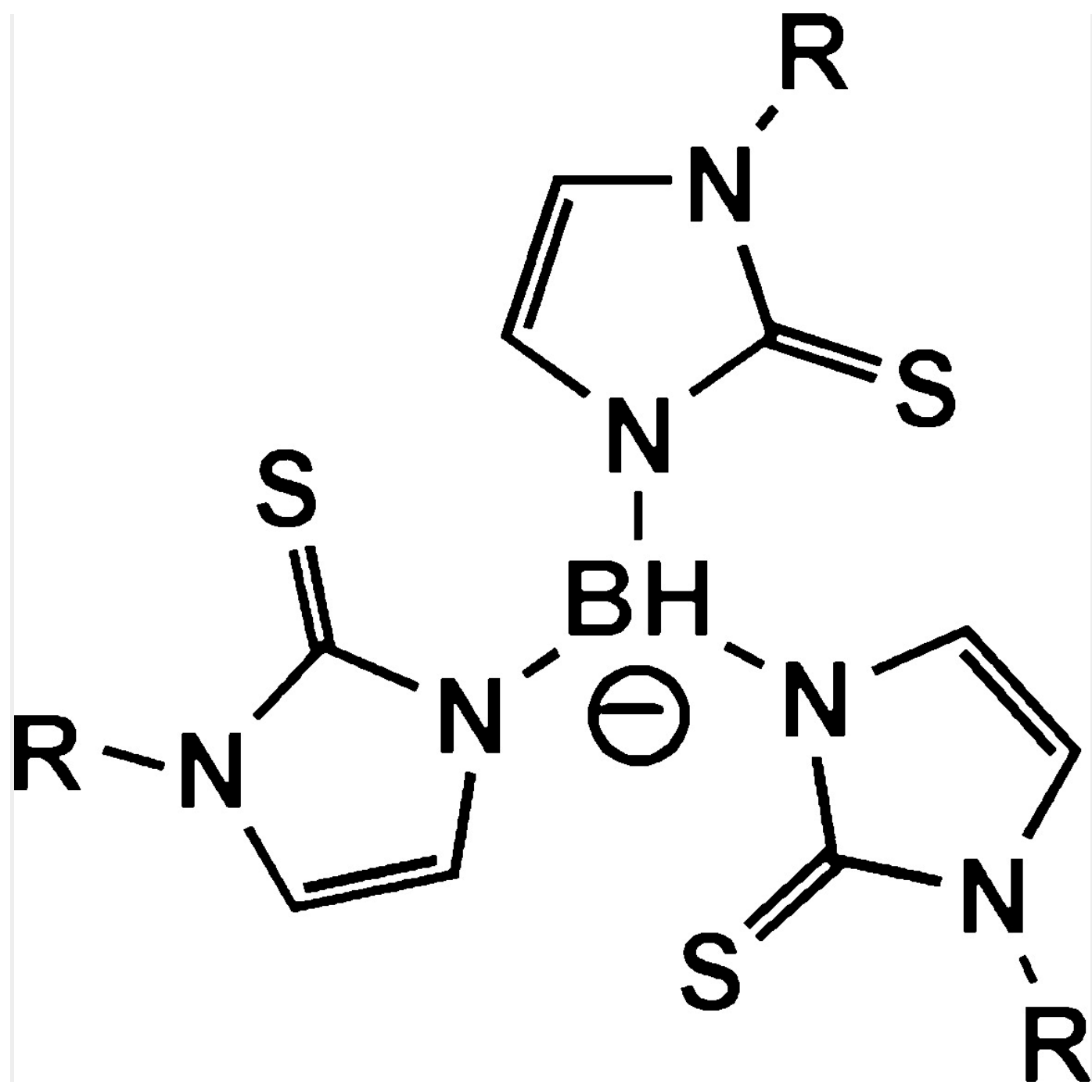

Figure 2. Tris(2-mercapto-1-R-imidazolyl)hydroborates, $\left[\mathrm{HB}\left(\mathrm{mim}^{\mathrm{R}}\right)_{3}\right]^{-}$or [ $\left[\mathrm{Tm}^{\mathrm{R}}\right]^{-}$-

We have been developing the chemistry of the tris(mercaptothiadiazolyl)borate ligand, $\left[\mathrm{HB}(\mathrm{mtda})_{3}\right]^{-}$, the socalled Janus scorpionate (Figure $\underline{3}$ ).(16) The Janus scorpionate can be considered a hybrid of Trofimenko's tris(pyrazolyl)borate $(1,2)$ and of Reglinski's tris(mercaptoimidazolyl)borate $(12)$ and is a close relative of Bailey's and Marchiò's ambidentate thioxotriazolylborate.(17) In every case examined to date, the heterocycles in this new Janus scorpionate adopt a conformation that places all soft sulfur donors on one face of the ligand directed toward the boron-hydride moiety, whereas all hard nitrogen donors are on the opposite face directed away from the hydride. This arrangement presumably minimizes electron repulsions between the lone pairs of electrons on the relatively large thione donors and the repulsive interactions between the thione groups and the $\pi$-clouds of the adjacent heterocycles. As might be expected, this ligand displays remarkable coordination versatility and metal-coordination capacity highlighted by the structure of the thallium( +1$)$ complex where the ligand, using only its sulfur donors, was found to bind five thallium cations in a remarkable $\mu_{5}-\left(\mathrm{K}^{2}, \mathrm{~K}^{2}, \mathrm{~K}^{2}, \mathrm{~K}^{1}, \mathrm{~K}^{1}-\mathrm{L}\right)$ binding mode.(16b) We have also demonstrated that the different donor sets $(N, N, N$ - or $S, S, S-)$ on either face of the Janus scorpionate allow for controlled organization of alkali metal and/or mixed alkali metal coordination polymers(16a) according to Pearson's hard-soft acid-base concept.(18) It was hoped that by constructing 
assemblies of metal ions connected by these electroactive bidentate ligands (the constituent heterocycles are exploited in battery applications(19) and show desirable surface adsorption properties(20)) new conducting materials with tunable properties could be developed. For this purpose, we began to explore the ligand's coordination chemistry with iron(II), given the propensity for Fe" $\mathrm{N}_{6}$ kernels to exhibit electronic/magnetic bistability. In particular, we were enticed with the prospect of developing new chemical switches and wanted to determine whether changes in binding pocket size (induced either by changing the steric demand of the $\mathrm{R}$ groups on the $\mathrm{HB}\left(\mathrm{mtda}^{\mathrm{R}}\right)_{3}$ ligands and or by the structural distortions that occur upon complexation of different size metal cations to the opposing faces of the ligand) could dictate the ground spin-state of iron(II) because high-spin iron(II) is effectively a larger cation than low-spin iron(II) (owing to population of $\mathrm{e}_{\mathrm{g}}{ }^{*}$ orbitals in the former). This contribution is the first to describe the preparation and properties of the second-generation methyl-substituted Janus derivatives $(\mathrm{M})\left[\mathrm{HB}\left(\mathrm{mtda}^{\mathrm{Me}}\right)_{3}\right]\left(\mathrm{M}=\mathrm{Na}, \mathrm{K}, \mathrm{NBu}_{4}\right)$. Justification for this simple ligand modification being termed second-generation is presented based on the discrepancies between the properties of the two iron complexes, $\mathrm{Fe}\left[\mathrm{HB}\left(\mathrm{mtda}^{\mathrm{R}}\right)_{3}\right]_{2}(\mathrm{R}=\mathrm{H}, \mathrm{Me})$, that are apparent from structural and spectroscopic characterization data, including a Mössbauer spectral study.

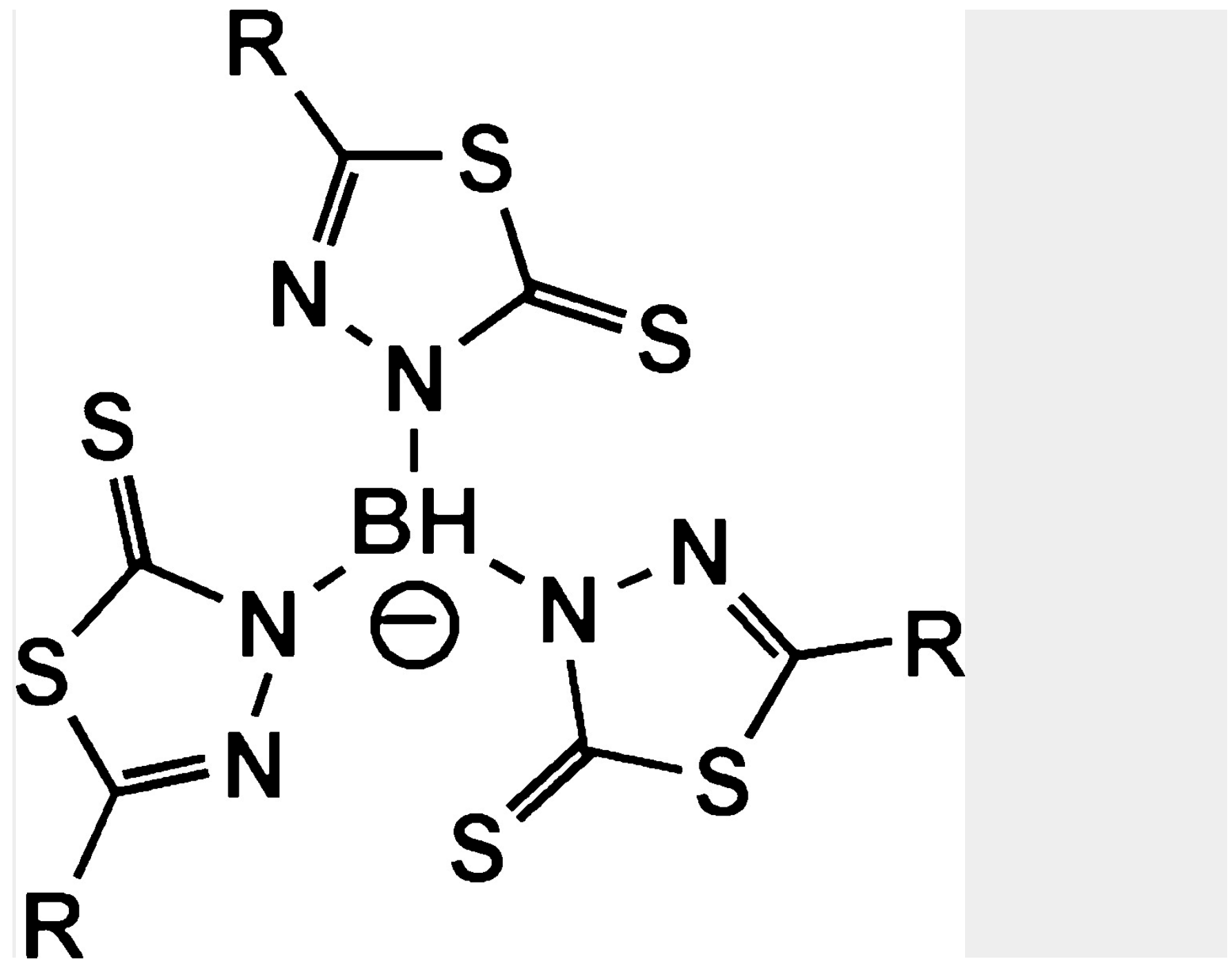

Figure 3. Janus scorpionate, tris(mercaptothiadiazolyl)borate, $\left[\mathrm{HB}\left(\mathrm{mtda}^{\mathrm{R}}\right)_{3}\right]^{-}$. 


\section{Experimental Section}

The compounds 2-mercapto-1,3,4-thiadiazole, $\mathrm{H}$ (mtda), and 2-mercapto-5-methyl-1,3,4-thiadiazole, $\mathrm{H}$ (mtdaMe), were obtained from Alfa Aesar, whereas all other reagents were obtained from Aldrich; all were used without further purification. Solvents were dried by conventional procedures and distilled prior to use, except where noted. The alkali metal borohydrides were stored and manipulated in a drybox with purified argon atmosphere. Most other manipulations were performed under nitrogen using Schlenk techniques, except where noted. The compounds (M) $\left[\mathrm{HB}(\mathrm{mtda})_{3}\right]\left(\mathrm{M}=\mathrm{Na}, \mathrm{K}, \mathrm{NBu}_{4}\right)$ were prepared as described earlier.(16) Midwest MicroLab, LLC, Indianapolis, Indiana 45250, performed all elemental analyses. Melting point determinations were made on samples contained in glass capillaries using an Electrothermal 9100 apparatus and are uncorrected. IR spectra of compounds in $\mathrm{KBr}$ pellets were recorded on a Nicolet Magna-IR 560 spectrometer. ${ }^{1} \mathrm{H}$ and ${ }^{13} \mathrm{C}$ NMR spectra were recorded on a Varian $300 \mathrm{MHz}$ spectrometer. Chemical shifts were referenced to solvent resonances at $\delta_{\mathrm{H}} 2.50$ and $\delta_{C} 39.51$ for DMSO, $\delta_{H} 2.05$ and $\delta_{C} 29.92$ for acetone- $d_{6}, \delta_{H} 4.80$ for $D_{2} O, \delta_{H} 2.75,2.92$ and $\delta_{H} 8.03$ and $\delta_{\mathrm{c}} 163.15$ for $\mathrm{dmf}-d_{7}$. Solid-state magnetic measurements were made at room temperature using an MK1 magnetic susceptibility balance.

\section{Syntheses}

$\mathrm{Na}[\mathrm{HB}(\mathrm{mtdaMe}) 3], 1$

A magnetically stirred neat mixture of $4.28 \mathrm{~g}(32.4 \mathrm{mmol}) \mathrm{H}\left(\mathrm{mtda}^{\mathrm{me}}\right)$ and $0.350 \mathrm{~g}(9.25 \mathrm{mmol}) \mathrm{NaBH}_{4}$ in a flask connected to a gas meter was heated by an external oil bath. Evolution of hydrogen gas commenced at about $120^{\circ} \mathrm{C}$ became vigorous upon heating to $140^{\circ} \mathrm{C}$ and stopped after $2 \mathrm{~h}$ at $185^{\circ} \mathrm{C}$. Care: The mixture decomposes if heated above $200^{\circ} \mathrm{C}$. The crude product mixture was cooled to room temperature, washed with three $20 \mathrm{~mL}$ portions of THF (to remove excess $\mathrm{H}\left(\mathrm{mtda}^{\mathrm{Me}}\right)$ ), followed by three $20 \mathrm{~mL}$ portions of $\mathrm{Et}_{2} \mathrm{O}$. After drying under vacuum, $2.86 \mathrm{~g}(72 \%)$ of 1 was obtained as a colorless powder. Mp, $196{ }^{\circ} \mathrm{C}$, dec brown-red. Anal. Calcd (Found) for $\mathrm{C}_{9} \mathrm{H}_{10} \mathrm{BN}_{6} \mathrm{NaS}_{6}$ : C, 25.23 (24.87), H, 2.35 (2.66), N, 19.62 (19.28). ${ }^{1 \mathrm{H}} \mathrm{NMR}\left(\delta, \mathrm{dmf}-d_{7}\right): 3.61\left(\mathrm{~s}, 9 \mathrm{H}, 3 \mathrm{CH}_{3}\right) ;{ }^{13} \mathrm{C}$ $\operatorname{NMR}\left(\delta, \mathrm{dmf}-d_{7}\right): 191.6(\mathrm{C}=\mathrm{S}), 154.7(C=\mathrm{N}), 15.9\left(\mathrm{CH}_{3}\right) . \mathrm{IR}\left(\mathrm{cm}^{-1}, \mathrm{KBr}\right): \mathrm{v}_{\mathrm{B}-\mathrm{H}}=2398$. X-ray quality colorless needles of a trimethanol solvate, $1.3 \mathrm{MeOH}$, were obtained by slowly cooling a hot methanol solution. Crystals dried under vacuum and then exposed to air had the following characterization data: Anal. Calcd (Found) for 1·MeOH$\cdot \mathrm{H}_{2} \mathrm{O}$ or $\mathrm{C}_{10} \mathrm{H}_{16} \mathrm{BN}_{6} \mathrm{NaO}_{2} \mathrm{~S}_{6}: \mathrm{C}, 25.10$ (24.73), $\mathrm{H}, 3.37$ (3.29), N, 17.56 (17.50). ${ }^{1 \mathrm{H}} \mathrm{NMR}$ (DMSO- $d_{6}$ ) $\delta_{\mathrm{H}} 4.09$ (q, $J=5.59 \mathrm{~Hz}, 2 \mathrm{H}$, $\mathrm{OH}), 3.33\left(\mathrm{~s}, 2 \mathrm{H}, \mathrm{H}_{2} \mathrm{O}\right), 3.17\left(\mathrm{~d}, J=5.59 \mathrm{~Hz}, 3 \mathrm{H}, \mathrm{OCH}_{3}\right), 2.30\left(\mathrm{~s}, 9 \mathrm{H}, \mathrm{mtda}-\mathrm{CH}_{3}\right) .{ }^{13} \mathrm{C}$ NMR $\left(\mathrm{DMSO}-d_{6}\right) \delta_{\mathrm{c}}, 189.9(\mathrm{C}=\mathrm{S})$, $154.2(\mathrm{C}=\mathrm{N}), 48.6\left(\mathrm{OCH}_{3}\right), 16.1\left(\mathrm{mtda}-\mathrm{CH}_{3}\right) . \mathrm{IR}\left(\mathrm{cm}^{-1}, \mathrm{KBr}\right): \mathrm{v}_{\mathrm{B}-\mathrm{H}}=2635$.

$\mathrm{K}[\mathrm{HB}(\mathrm{mtdaMe}) 3], 2$

A magnetically stirred neat mixture of $4.28 \mathrm{~g}(32.4 \mathrm{mmol}) \mathrm{H}$ (mtdaмe) and $0.500 \mathrm{~g}(9.27 \mathrm{mmol}) \mathrm{KBH}_{4}$ in a flask connected to a gas meter was heated by an external oil bath. Evolution of hydrogen gas commenced at about $100{ }^{\circ} \mathrm{C}$, became vigorous upon heating to $115^{\circ} \mathrm{C}$, and stopped after $2 \mathrm{~h}$ at $205^{\circ} \mathrm{C}$. The crude product mixture was cooled to room temperature, washed with three $20 \mathrm{~mL}$ portions of THF (to remove excess $\mathrm{H}$ (mtdaмe)), followed by three $20 \mathrm{~mL}$ portions of $\mathrm{Et}_{2} \mathrm{O}$. After drying under vacuum, $2.65 \mathrm{~g}$ (64\%) of 2 was obtained as a colorless powder. Mp, $226-227^{\circ} \mathrm{C}$. Anal. Calcd (Found) for $\mathrm{C}_{9} \mathrm{H}_{10} \mathrm{BKN}_{6} \mathrm{~S}_{6}: \mathrm{C}, 24.32$ (23.97); H, 2.27 (2.50); N, 18.91 (18.66). ${ }^{1 \mathrm{H}}$ NMR (DMSO- $\left.d_{6}\right) \delta_{\mathrm{H}}, 2.30\left(\mathrm{~s}, 9 \mathrm{H}, \mathrm{CH}_{3}\right) .{ }^{13} \mathrm{C}$ NMR (DMSO- $\left.d_{6}\right) \delta_{\mathrm{c}}, 189.93(\mathrm{C}=\mathrm{S}), 153.9(\mathrm{C}=\mathrm{N}), 15.9\left(\mathrm{CH}_{3}\right) . \mathrm{IR}$ $\left(\mathrm{cm}^{-1}, \mathrm{KBr}\right): \mathrm{V}_{\mathrm{B}-\mathrm{H}}=2644$. X-ray quality colorless block crystals of a methanol solvate $\mathrm{K}_{2}\left[\mathrm{HB}\left(\mathrm{mtda}^{\mathrm{Me}}\right)_{3}\right]_{2} \cdot 3 \mathrm{MeOH}, \mathbf{2} \cdot 1.5 \mathrm{MeOH}$, were obtained by slowly cooling a hot supersaturated methanol solution. The crystals lose methanol when drying under vacuum to give a substance that analyzed as $2 \cdot 0.5 \mathrm{MeOH}$ Anal. Calcd (Found) for $\mathrm{C}_{9.5} \mathrm{H}_{12} \mathrm{BKN}_{6} \mathrm{O}_{0.5} \mathrm{~S}_{6}: \mathrm{C}, 24.78$ (25.01), H, 2.63 (2.69), N, 18.91 (18.58). 
(NBu4)[HB(mtdaMe)3], 3

A biphasic mixture of $2.00 \mathrm{~g}(6.20 \mathrm{mmol})$ of $\left(\mathrm{NBu}_{4}\right) \mathrm{Br}$ in $25 \mathrm{~mL}$ of $\mathrm{CH}_{2} \mathrm{Cl}_{2}$ and $2.66 \mathrm{~g}(6.21 \mathrm{mmol}) \mathrm{Na}\left[\mathrm{HB}(\mathrm{mtda})_{3}\right]$ (1) in $25 \mathrm{~mL}$ water was vigorously stirred at room temperature for $2 \mathrm{~h}$ and the layers were separated. The organic layer was washed with $25 \mathrm{~mL} \mathrm{H}_{2} \mathrm{O}$ and separated. After three more such washings and separations, the organic layer was dried over $\mathrm{MgSO}_{4}$, filtered, and solvent was removed under vacuum to give $3.05 \mathrm{~g}$ (76\%) 3 as a colorless powder. $\mathrm{Mp}, 174-175{ }^{\circ} \mathrm{C}$. Anal. Calcd (Found) for $\mathrm{C}_{25} \mathrm{H}_{46} \mathrm{BN}_{7} \mathrm{~S}_{6}$ : C, 46.35 (46.01), H, 7.16 (7.32), N, 15.13 (14.87). ${ }^{1 \mathrm{H}} \mathrm{NMR}\left(\mathrm{DMSO}-d_{6}\right) \delta_{\mathrm{H}}, 3.16\left(\mathrm{t}, J=8.30 \mathrm{~Hz}, 8 \mathrm{H}, \mathrm{N}-\mathrm{CH}_{2}\right), 2.30\left(\mathrm{~s}, 9 \mathrm{H}, \mathrm{mtda}-\mathrm{CH}_{3}\right), 1.56\left(\mathrm{~m}, 8 \mathrm{H}, \mathrm{CH}_{2}\right), 1.31(\mathrm{~m}$, $\left.8 \mathrm{H}, \mathrm{CH}_{2}\right), 0.93\left(\mathrm{t}, J=7.34 \mathrm{~Hz}, 12 \mathrm{H}, \mathrm{CH}_{3}\right) .{ }^{13} \mathrm{C} \mathrm{NMR}\left(\mathrm{DMSO}-d_{6}\right) \delta_{\mathrm{c}}, 189.7(\mathrm{C}=\mathrm{S}), 153.9\left(\mathrm{CH}_{3} \mathrm{C}=\mathrm{N}\right), 57.5\left(\mathrm{~N}-\mathrm{CH}_{2}\right), 23.1$ $\left.\mathrm{CH}_{2}\right), 19.2\left(\mathrm{CH}_{2}\right), 15.9\left(\mathrm{mtda}-\mathrm{CH}_{3}\right), 13.5\left(\mathrm{CH}_{3}\right) .{ }^{1} \mathrm{H} \mathrm{NMR}\left(\mathrm{CDCl}_{3}\right) \delta_{\mathrm{H}} 3.32\left(\mathrm{~m}, 8 \mathrm{H}, \mathrm{NCH}_{2}\right), 2.35\left(\mathrm{~s}, 9 \mathrm{H}, \mathrm{mtda}-\mathrm{CH}_{3}\right), 1.64$ $\left(\mathrm{m}, 8 \mathrm{H}, \mathrm{CH}_{2}\right), 1.39\left(\mathrm{~m}, 8 \mathrm{H}, \mathrm{CH}_{2}\right), 0.94\left(\mathrm{~m}, 12 \mathrm{H}, \mathrm{CH}_{3}\right) .{ }^{13} \mathrm{C} \mathrm{NMR}\left(\delta, \mathrm{CDCl}_{3}\right): 191.2(\mathrm{C}=\mathrm{S}), 156.3(\mathrm{HC}=\mathrm{N}), 59.1\left(\mathrm{CH}_{3}\right)$, $24.6\left(\mathrm{CH}_{2}\right), 19.9\left(\mathrm{CH}_{2}\right), 16.9\left(\mathrm{CH}_{2}\right), 13.9\left(\mathrm{CH}_{3}\right) . \mathrm{IR}\left(\mathrm{cm}^{-1}, \mathrm{KBr}\right): \mathrm{v}_{\mathrm{B}-\mathrm{H}}=2518$.

\section{$\mathrm{Fe}[\mathrm{HB}(\mathrm{mtda}) 3] 2,4$}

A solution of $1.53 \mathrm{~g}(2.53 \mathrm{mmol})$ of $\left(\mathrm{NBu}_{4}\right)\left[\mathrm{HB}(\mathrm{mtda})_{3}\right]$ in $20 \mathrm{~mL}$ of THF was added to a THF solution $(20 \mathrm{~mL})$ of $0.430 \mathrm{~g}(1.27 \mathrm{mmol})\left[\mathrm{Fe}\left(\mathrm{H}_{2} \mathrm{O}\right)_{6}\right]\left(\mathrm{BF}_{4}\right)_{2}$. The resulting solution turned to bright yellow on mixing, and after a few seconds a pink solid precipitated. After stirring $30 \mathrm{~min}$, the pink solid was collected by filtration, washed sequentially with $10 \mathrm{~mL}$ THF and two $10 \mathrm{~mL}$ portions $\mathrm{Et}_{2} \mathrm{O}$, and dried by heating under vacuum for $4-6 \mathrm{~h}$ to give $0.853 \mathrm{~g}(86 \%)$ of $4 . \mathrm{Mp}, 218{ }^{\circ} \mathrm{C} \mathrm{dec}$. Anal. Calcd (found) for $\mathrm{C}_{12} \mathrm{H}_{8} \mathrm{~B}_{2} \mathrm{FeN}_{12} \mathrm{~S}_{12}: \mathrm{C}, 18.42$ (18.27), $\mathrm{H}, 1.03$ (0.98), $\mathrm{N}$ 21.48 (21.65). ${ }^{1} \mathrm{H}$ NMR (DMSO- $\left.d_{6}\right) \delta_{\mathrm{H}}, 8.52\left(\mathrm{~s}, 6 \mathrm{H}, \mathrm{HC}=\mathrm{N}\right.$ ). ${ }^{13} \mathrm{C}$ NMR (DMSO- $\left.d_{6}\right) \delta_{c}, 185.8$ (C=S), 142.2 (HC=N). IR $\left(\mathrm{cm}^{-1}, \mathrm{KBr}\right): \mathrm{v}_{\mathrm{B}-\mathrm{H}}=2522 \mathrm{~cm}^{-1}$. If the sample is not heated under vacuum for several hours, THF and $\mathrm{H}_{2} \mathrm{O}$ is tenaciously held. For example, when a sample from a separate experiment was held under vacuum for $2 \mathrm{~h}$ without heating, it analyzed as $4 \cdot 2 \mathrm{THF} \cdot 1.5 \mathrm{H}_{2} \mathrm{O}$. Anal. Calcd (found) for $\mathrm{C}_{20} \mathrm{H}_{27} \mathrm{~B}_{2} \mathrm{FeN}_{12} \mathrm{O}_{3.5} \mathrm{~S}_{12}: \mathrm{C}, 25.19$ (24.76), $\mathrm{H}$, 2.85 (2.83), $\mathrm{N} 17.62$ (17.70). Pink crystals of 4.4solvent (solvent $=\mathrm{CH}_{3} \mathrm{CN}$ or DMF) can be grown by slow diffusion of $\mathrm{Et}_{2} \mathrm{O}$ into solutions of the iron complex in the appropriate solvent.

$\mathrm{Fe}(\mathrm{H} 2 \mathrm{O}) 4[\mathrm{HB}(\mathrm{mtda}) 3] 2,5$

A solution of $0.460 \mathrm{~g}(1.14 \mathrm{mmol})$ of $\mathrm{K}\left[\mathrm{HB}(\mathrm{mtda})_{3}\right]$ in $3 \mathrm{~mL} \mathrm{H}{ }_{2} \mathrm{O}$ was added to a solution of $0.050 \mathrm{~g}(0.394 \mathrm{mmol})$ $\mathrm{FeCl}_{2}$ in $8 \mathrm{~mL} \mathrm{H} \mathrm{O}$, initially affording a pale-green solution that within minutes deposits a colorless solid. After stirring $30 \mathrm{~min}$, the solid was collected by filtration, washed with $20 \mathrm{~mL} \mathrm{Et}_{2} \mathrm{O}$, and dried under vacuum to leave $0.273 \mathrm{~g}(81 \%) 5$ as a bright yellow solid. $\mathrm{Mp}, 170{ }^{\circ} \mathrm{C}$, dec. Anal. Calcd (found) for $\mathrm{C}_{12} \mathrm{H}_{16} \mathrm{~B}_{2} \mathrm{FeN}_{12} \mathrm{O}_{4} \mathrm{~S}_{12}$ : $\mathrm{C}, 16.87$ (17.27); H, 1.89, (1.98) 9; N, 19.67 (19.35). ${ }^{1} \mathrm{H}$ NMR ( $\left.\delta, \mathrm{DMSO}\right): 8.46(\mathrm{~s}, 6 \mathrm{H}, \mathrm{HC}=\mathrm{N}), 4.82\left(\mathrm{~b}, 8 \mathrm{H}, 4 \mathrm{H}_{2} \mathrm{O}\right) .{ }^{13} \mathrm{C} N M R$

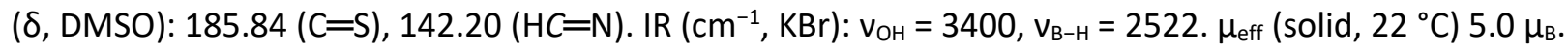
Alternatively, this yellow compound can be prepared quantitatively by dissolving pink $\mathrm{Fe}\left[\mathrm{HB}\left(\mathrm{mtda}_{3}\right)_{2}\right](4)$ in water and removing solvent by vacuum distillation. Single crystals of $\left[\mathrm{Fe}\left(\mathrm{H}_{2} \mathrm{O}\right)_{6}\right]\left[\mathrm{HB}(\mathrm{mtda})_{3}\right]_{2} \cdot 6 \mathrm{H}_{2} \mathrm{O}\left(6 \cdot 6 \mathrm{H}_{2} \mathrm{O}\right)$ suitable for an $\mathrm{X}$-ray diffraction study can be obtained by allowing an aqueous solution of either $\mathrm{Fe}\left[\mathrm{HB}\left(\mathrm{mtda}_{3}\right)_{2}\right]$ (4) or $\mathrm{Fe}\left(\mathrm{H}_{2} \mathrm{O}\right)_{4}\left[\mathrm{HB}(\mathrm{mtda})_{3}\right]_{2}(5)$ to slowly evaporate for over a month. Analysis of an air-dried crystalline sample of $6 \cdot 6 \mathrm{H}_{2} \mathrm{O}$ showed loss of four water molecules to give $\left[\mathrm{Fe}\left(\mathrm{H}_{2} \mathrm{O}\right)_{6}\right]\left[\mathrm{HB}(\mathrm{mtda})_{3}\right]_{2} \cdot 2 \mathrm{H}_{2} \mathrm{O}\left(6 \cdot 2 \mathrm{H}_{2} \mathrm{O}\right)$. Anal. Calcd (found) for $\mathrm{C}_{12} \mathrm{H}_{18} \mathrm{~B}_{2} \mathrm{FeN}_{12} \mathrm{~S}_{12} \mathrm{O}_{6}$ : C, 15.55 (15.95), H, 2.39 (2.32), N 18.14 (17.89). Similarly crystals of [Fe(DMSO $\left.)_{6}\right]\left[\mathrm{HB}(\mathrm{mtda})_{3}\right]_{2} \cdot 2 \mathrm{CHCl}_{3}\left(7 \cdot 2 \mathrm{CHCl}_{3}\right)$ were grown by dissolving 4 in $\mathrm{DMSO}$ and layering onto $\mathrm{CHCl}_{3}$, and allowing the layers to diffuse.

$\mathrm{Fe}[\mathrm{HB}(\mathrm{mtdaMe}) 3] 2,8$

Method A.

A solution of $0.100 \mathrm{~g}(0.296 \mathrm{mmol})$ of $\left[\mathrm{Fe}\left(\mathrm{H}_{2} \mathrm{O}\right)_{6}\right]\left(\mathrm{BF}_{4}\right)_{2}$ in $5 \mathrm{~mL} \mathrm{CH} \mathrm{CNN}_{3}$ was added to a solution of $0.263 \mathrm{~g}(0.592$ mmol) of $\mathrm{K}\left[\mathrm{HB}\left(\mathrm{mtda}^{\mathrm{Me}}\right)_{3}\right](2)$ in $10 \mathrm{~mL}$ of $\mathrm{CH}_{3} \mathrm{CN}$, whereupon a golden-yellow solid immediately precipitated. 
After stirring $30 \mathrm{~min}$ at room temperature, the solid was collected by filtration, washed with three $15 \mathrm{~mL}$ portions of $\mathrm{CH}_{3} \mathrm{CN}$, and was dried by heating to $60^{\circ} \mathrm{C}$ (external oil bath) under vacuum for several hours to leave $0.232 \mathrm{~g}(91 \%) 8$ as a golden-yellow solid. $\mathrm{Mp}, 228^{\circ} \mathrm{C}$ dec to brown solid. Anal. Calcd (found) for $\mathrm{C}_{18} \mathrm{H}_{20} \mathrm{~B}_{2} \mathrm{FeN}_{12} \mathrm{~S}_{12}$ : C, 24.95 (25.30), H, 2.33 (2.07), N 19.39 (19.61). ${ }^{1} \mathrm{H}$ NMR (DMSO- $d_{6}$ ) $\delta_{\mathrm{H}}, 2.24$ (s, $\left.\mathrm{CH}_{3}\right) .{ }^{13} \mathrm{C}$ NMR ( $\delta$, DMSO): 185.98 $(\mathrm{C}=\mathrm{S}), 150.48(\mathrm{C}=\mathrm{N}), 12.41\left(\mathrm{CH}_{3}\right) . \mathrm{IR}\left(\mathrm{cm}^{-1}, \mathrm{KBr}\right): \mathrm{v}_{\mathrm{B}-\mathrm{H}}=2512 . \mu_{\text {eff }}\left(\right.$ solid, $\left.22^{\circ} \mathrm{C}\right) 5.1 \mu_{\mathrm{B}}$.

\section{Method B}

A solution of $0.500 \mathrm{~g}(0.772 \mathrm{mmol})\left(\mathrm{NBu}_{4}\right)\left[\mathrm{HB}\left(\mathrm{mtdame}_{3}\right](3)\right.$ in $10 \mathrm{~mL}$ THF was added to a solution of $0.130 \mathrm{~g}$ $(0.385 \mathrm{mmol})\left[\mathrm{Fe}\left(\mathrm{H}_{2} \mathrm{O}\right)_{6}\right]\left(\mathrm{BF}_{4}\right)_{2}$ in $30 \mathrm{~mL}$ THF where a golden-yellow solid immediately precipitated. After stirring $30 \mathrm{~min}$ at room temperature, the solid was collected by filtration, washed sequentially with three $20 \mathrm{~mL}$ portions of THF and three $20 \mathrm{~mL}$ portions $\mathrm{Et}_{2} \mathrm{O}$, and was dried by heating to $60^{\circ} \mathrm{C}$ (external oil bath) under vacuum for several hours to afford $0.210 \mathrm{~g}(63 \%) 7$ as a golden-yellow solid with characterization data identical to above. Note: heating under vacuum is necessary to completely remove solvent. A sample obtained from separate experiment where the solid was only dried under vacuum $2 \mathrm{~h}$ at room temperature analyzed as 8-0.5THF. Anal. Calcd (found) for $\mathrm{C}_{20} \mathrm{H}_{24} \mathrm{~B}_{2} \mathrm{FeN}_{12} \mathrm{O}_{0.5} \mathrm{~S}_{12}$ : C, 26.61 (26.78), H, 2.68 (2.78), N 18.62 (18.33). Yellow crystals of $\mathrm{Fe}(\mathrm{DMF})_{4}\left[\mathrm{HB}\left(\mathrm{mtda}^{\mathrm{Me}}\right)_{3}\right]_{2}(\mathbf{9})$ and $\left[\mathrm{Fe}(\mathrm{DMF})_{6}\right]\left[\mathrm{HB}\left(\mathrm{mtda}^{\mathrm{Me}}\right)_{3}\right]_{2} \cdot 2 \mathrm{DMF}(\mathbf{1 0} \cdot 2 \mathrm{DMF})$ were grown by slow evaporation (over a week) of DMF from a solution of $\mathbf{8}$ in DMF. The relative distribution of $\mathbf{9}$ to $10 \cdot 2 \mathrm{DMF}$ appears capricious.

\section{Crystallography}

X-ray intensity data from a colorless prism of $\mathrm{Na}[\mathrm{HB}(\mathrm{mtdaMe}) 3] \cdot 3 \mathrm{MeOH}(1 \cdot 3 \mathrm{MeOH})$, a colorless block of $\mathrm{K} 2[\mathrm{HB}(\mathrm{mtdaMe}) 3] 2 \cdot 3 \mathrm{MeOH}(2 \cdot 1.5 \mathrm{MeOH})$, a pink prism of Fe[HB(mtda)3]2·4DMF (4·4DMF), a pale-orange block of [Fe(DMSO)6][HB(mtda)3]2 $2 \mathrm{CHCl} 3(7 \cdot 2 \mathrm{CHCl} 3)$, a pale-yellow block of Fe(DMF)4[HB(mtdaMe)3]2 (9), and a yellow prism of [Fe(DMF)6][HB(mtdaMe)3]2-2DMF (10.2DMF) were measured at 100(2) K with a Bruker AXS 3circle diffractometer equipped with a SMART2[S10] CCD detector using $\mathrm{Cu}(\mathrm{K} \alpha)$ radiation, whereas data from a small irregular purple crystal of $\mathrm{Fe}[\mathrm{HB}(\mathrm{mtda}) 3] 2 \cdot 4 \mathrm{CH} 3 \mathrm{CN}(4 \cdot 4 \mathrm{CH} 3 \mathrm{CN})$, and of a colorless block of [Fe(H2O)6][HB(mtda)3]2.6H2O (6.6H2O) were measured at 150(1) $\mathrm{K}$ on a Bruker SMART APEX diffractometer (Mo K $\alpha$ radiation, $\lambda=0.71073 \AA$ )..(21) Raw data frame integration and Lp corrections were performed with SAINT+.(21) Final unit cell parameters were determined by least-squares refinement of 4477 reflections from the data set of $1.3 \mathrm{MeOH}$, of 7919 reflections from that of $2 \cdot 1.5 \mathrm{MeOH}, 5573$ reflections from that of $4.4 \mathrm{DMF}, 1280$ reflections of $4.4 \mathrm{CH} 3 \mathrm{CN}, 6639$ reflections from that of $6 \cdot 6 \mathrm{H} 2 \mathrm{O}, 5231$ reflections from that of $7 \cdot 2 \mathrm{CHCl} 3,8609$ reflections from that of 9 , and 5842 reflections from that of $10 \cdot 2 \mathrm{DMF}$, with I $>2 \sigma(\mathrm{I})$ for each. Analysis of the data showed negligible crystal decay during collection in each case. Direct methods structure solutions, difference Fourier calculations and full-matrix least-squares refinements against F2 were performed with SHELXTL.(22) Numerical absorption corrections were applied to $2 \cdot 1.5 \mathrm{MeOH}, 7 \cdot 2 \mathrm{CHCl} 3$ and 9 , whereas no absorption correction was applied to the data for either $4.4 \mathrm{CH} 3 \mathrm{CN}$ or $6 \cdot 6 \mathrm{H} 2 \mathrm{O}$. Semiempirical absorption correction based on the multiple measurement of equivalent reflections was applied to the data of each of the remainder of compounds with SADABS.(21)All non-hydrogen atoms were refined with anisotropic displacement parameters. Hydrogen atoms were either refined freely or treated as riding atoms (Supporting Information for 
details). The X-ray crystallographic parameters and further details of data collection and structure refinements are presented in Tables $\underline{\underline{1}}$ and $\underline{2}$.

Table 1. Crystallographic Data Collection and Structure Refinement for $\mathrm{Na}\left[\mathrm{HB}\left(\mathrm{mtda}^{\mathrm{Me}}\right)_{3}\right] \cdot 3 \mathrm{MeOH}(1 \cdot 3 \mathrm{MeOH})$, $\mathrm{K}_{2}\left[\mathrm{HB}\left(\mathrm{mtda}^{\mathrm{Me}}\right)_{3}\right]_{2} \cdot 3 \mathrm{MeOH}(2 \cdot 1.5 \mathrm{MeOH}), \mathrm{Fe}\left[\mathrm{HB}(\mathrm{mtda})_{3}\right]_{2} \cdot 4 \mathrm{DMF}(4 \cdot 4 \mathrm{DMF})$, and $\mathrm{Fe}\left[\mathrm{HB}(\mathrm{mtda})_{3}\right]_{2} \cdot 4 \mathrm{CH}_{3} \mathrm{CN}\left(4 \cdot 4 \mathrm{CH}_{3} \mathrm{CN}\right)$

\begin{tabular}{|c|c|c|c|c|}
\hline compound & $1 \cdot 3 \mathrm{MeOH}$ & $\mathbf{2 \cdot 1 . 5 \mathrm { MeOH }}$ & 4.4DMF & $4 \cdot 4 \mathrm{CH}_{3} \mathrm{CN}$ \\
\hline formula & $\mathrm{C}_{12} \mathrm{H}_{22} \mathrm{BN}_{6} \mathrm{NaO}_{3} \mathrm{~S}_{6}$ & $\mathrm{C}_{10.5} \mathrm{H}_{16} \mathrm{BKN}_{6} \mathrm{O}_{1.5} \mathrm{~S}_{6}$ & $\mathrm{C}_{24} \mathrm{H}_{36} \mathrm{~B}_{2} \mathrm{FeN}_{16} \mathrm{O}_{4} \mathrm{~S}_{12}$ & $\mathrm{C}_{20} \mathrm{H}_{20} \mathrm{~B}_{2} \mathrm{FeN}_{16} \mathrm{~S}_{12}$ \\
\hline $\mathrm{fw}$ & 524.52 & 492.56 & 1074.88 & 946.71 \\
\hline cryst syst & orthorhombic & triclinic & monoclinic & monoclinic \\
\hline space group & Pbcm & $P I$ & $P 2_{1} / n$ & $P 2_{1} / n$ \\
\hline temperature $[\mathrm{K}]$ & $100(2)$ & $100(2)$ & $100(2)$ & $150(1)$ \\
\hline$a[\AA]$ & $11.0116(3)$ & $8.13750(10)$ & $9.0094(4)$ & $13.6645(10)$ \\
\hline$b[\AA ̊]$ & $15.8601(4)$ & $15.78230(10)$ & $13.4664(5)$ & $9.7325(7)$ \\
\hline$c[\AA]$ & $13.7977(4)$ & $17.8552(2)$ & $18.7165(7)$ & $13.9690(10)$ \\
\hline$\alpha[\mathrm{deg}]$ & 90 & $65.4420(10)$ & 90 & 90 \\
\hline$\beta[\mathrm{deg}]$ & 90 & $81.1870(10)$ & $97.652(2)$ & $91.592(2)$ \\
\hline$Y[\mathrm{deg}]$ & 90 & $87.5990(10)$ & 90 & 90 \\
\hline$V\left[\AA^{3}\right]$ & 2409.70(11) & $2060.45(4)$ & $2250.54(16)$ & $1857.0(2)$ \\
\hline$z$ & 4 & 2 & 2 & 2 \\
\hline$D_{\text {calcd }}\left[\mathrm{g} \mathrm{cm}^{-3}\right]$ & 1.446 & 1.588 & 1.586 & 1.693 \\
\hline$\lambda[\AA ⿻$ & 1.54178 a & 1.54178 & 1.54178 & $0.71073^{\underline{b}}$ \\
\hline$\mu\left[\mathrm{mm}^{-1}\right]$ & 5.651 & 8.097 & 8.344 & 1.125 \\
\hline abs. correction & multiscan & numerical & multiscan & none \\
\hline$F(000)$ & 1088 & 1012 & 1104 & 960 \\
\hline range [deg] & 4.89 to 60.95 & 2.75 to 67.33 & 4.06 to 61.51 & 2.06 to 22.54 \\
\hline reflns collected & 9835 & 17370 & 18623 & 13502 \\
\hline independent reflections & $1861\left(R_{\text {int }} 0.0322\right)$ & $6845\left(R_{\text {int }} 0.0226\right)$ & $3436\left(R_{\text {int }} 0.0396\right)$ & $2439\left(R_{\text {int }}=0.1155\right)$ \\
\hline$T(\min / \max )$ & $0.1099 / 0.7279$ & $0.1444 / 0.4433$ & $0.1351 / 0.3675$ & \\
\hline data/restraints/params & $1861 / 0 / 196$ & $6845 / 0 / 602$ & $3436 / 24 / 323$ & $2439 / 0 / 234$ \\
\hline GOF on $F^{2}$ & 0.975 & 0.993 & 1.028 & 1.018 \\
\hline$R 1 / w R 2[(I)] \subseteq$ & $0.0268 / 0.0696$ & $0.0264 / 0.0611$ & $0.0265 / 0.0659$ & $0.0483 / 0.0817$ \\
\hline$R 1 / w R 2$ (all data) & $0.0303 / 0.0715$ & $0.0305 / 0.0627$ & $0.0277 / 0.0664$ & $0.0825 / 0.0908$ \\
\hline
\end{tabular}

a Cu Ka.

b Mo Ka.

c $R 1=\sum\left\|F_{\mathrm{o}}|-| F\right\| / \Sigma\left|F_{\mathrm{o}}\right| w R 2=\left[\sum w\left(\left|F_{\mathrm{o}}\right|-\left|F_{\mathrm{c}}\right|\right)^{2} / \sum w\left|F_{\mathrm{o}}\right|^{2}\right]^{1 / 2}$.

Table 2. Crystallographic Data Collection and Structure Refinement for $\left[\mathrm{Fe}\left(\mathrm{H}_{2} \mathrm{O}\right)_{6}\right]\left[\mathrm{HB}(\mathrm{mtda})_{3}\right]_{2} \cdot 6 \mathrm{H}_{2} \mathrm{O}\left(6 \cdot 6 \mathrm{H}_{2} \mathrm{O}\right)$, $\left[\mathrm{Fe}(\mathrm{DMSO})_{6}\right]\left[\mathrm{HB}(\mathrm{mtda})_{3}\right]_{2} \cdot 2 \mathrm{CHCl}_{3}\left(7 \cdot 2 \mathrm{CHCl}_{3}\right), \mathrm{Fe}(\mathrm{DMF})_{4}\left[\mathrm{HB}\left(\mathrm{mtdaMe}_{3}\right]_{2}(9)\right.$, and $\left[\mathrm{Fe}(\mathrm{DMF})_{6}\right]\left[\mathrm{HB}\left(\mathrm{mtda}^{\mathrm{Me}}\right)_{3}\right]_{2} \cdot 2 \mathrm{DMF}$ (10.2DMF)

\begin{tabular}{lllll|}
\hline compound & $\mathbf{6} \cdot 6 \mathrm{H}_{2} \mathrm{O}$ & $\mathbf{7} \cdot 2 \mathrm{CHCl}_{3}$ & $\mathbf{9}$ & $\mathbf{1 0} \cdot 2 \mathrm{DMF}$ \\
formula & $\mathrm{C}_{12} \mathrm{H}_{32} \mathrm{~B}_{2} \mathrm{FeN}_{12} \mathrm{O}_{12} \mathrm{~S}_{12}$ & $\mathrm{C}_{26} \mathrm{H}_{46} \mathrm{~B}_{2} \mathrm{Cl}_{6} \mathrm{FeN}_{12} \mathrm{O}_{6} \mathrm{~S}_{18}$ & $\mathrm{C}_{30} \mathrm{H}_{48} \mathrm{~B}_{2} \mathrm{FeN}_{16} \mathrm{O}_{4} \mathrm{~S}_{12}$ & $\mathrm{C}_{42} \mathrm{H}_{76} \mathrm{~B}_{2} \mathrm{FeN}_{20} \mathrm{O}_{8} \mathrm{~S}_{12}$ \\
$\mathrm{fw}$ & 998.69 & 1490.00 & 1159.03 & 1451.42 \\
cryst syst & triclinic & triclinic & triclinic & triclinic \\
\hline
\end{tabular}




\begin{tabular}{|c|c|c|c|c|}
\hline space group & $P I$ & $P I$ & $P I$ & $P I$ \\
\hline temperature $[\mathrm{K}]$ & $150(1)$ & $100(2)$ & $100(2)$ & $100(2)$ \\
\hline$a[\AA]$ & $7.6358(5)$ & $12.0552(2)$ & $8.71280(10)$ & $11.3576(10)$ \\
\hline$b[\AA]$ & $11.7442(8)$ & $12.4970(2)$ & $11.55350(10)$ & $12.2918(11)$ \\
\hline$c[\AA]$ & $12.4548(8)$ & $13.3489(2)$ & $12.99550(10)$ & $13.7064(12)$ \\
\hline$\alpha[\mathrm{deg}]$ & $115.9180(10)$ & $116.0080(10)$ & $84.9810(10)$ & $67.851(5)$ \\
\hline$\beta[\mathrm{deg}]$ & $98.7860(10)$ & $94.4850(10)$ & $81.0120(10)$ & $72.618(4)$ \\
\hline$\gamma[\mathrm{deg}]$ & $90.2380(10)$ & $115.0470(10)$ & $81.0080(10)$ & $87.808(6)$ \\
\hline$V\left[\AA^{3}\right]$ & $989.61(11)$ & $1549.09(4)$ & $1273.56(2)$ & $1685.4(3)$ \\
\hline$Z$ & 1 & 1 & 1 & 1 \\
\hline$D_{\text {calcd }}\left[\mathrm{g} \mathrm{cm}^{-3}\right]$ & 1.676 & 1.597 & 1.511 & 1.430 \\
\hline$\lambda[\AA]$ & $0.71073 \underline{b}$ & 1.54178 & $1.54178 \mathrm{a}$ & $1.54178 \mathrm{a}$ \\
\hline$\mu\left[\mathrm{mm}^{-1}\right]$ & 1.078 & 10.409 & 7.415 & 5.782 \\
\hline abs. correction & none & numerical & numerical & multiscan \\
\hline$F(000)$ & 512 & 760 & 600 & 760 \\
\hline range [deg] & 1.85 to 26.42 & 3.89 to 67.17 & 3.45 to 67.12 & 3.66 to 61.44 \\
\hline reflns collected & 12037 & 12780 & 10611 & 14101 \\
\hline independent reflns & $4063\left(R_{\text {int }}=0.0377\right)$ & $5074\left(R_{\text {int }} 0.0194\right)$ & $4203\left(R_{\text {int }}=0.0147\right)$ & $4952\left(R_{\text {int }}=0.0279\right)$ \\
\hline$T(\min / \max )$ & & $0.0891 / 0.2300$ & $0.1467 / 0.3487$ & $0.3628 / 0.7609$ \\
\hline data/restraints/params & $4063 / 30 / 290$ & $5074 / 0 / 415$ & $4203 / 0 / 392$ & $4952 / 0 / 537$ \\
\hline GOF on $F^{2}$ & 1.008 & 1.023 & 1.040 & 1.044 \\
\hline$R 1 / w R 2[(I)>2 \sigma(I)] \underline{c}$ & $0.0266 / 0.0657$ & $0.0221 / 0.0553$ & $0.0226 / 0.0585$ & $0.0292 / 0.0717$ \\
\hline$R 1 / w R 2$ (all data) & $0.0301 / 0.0672$ & $0.0223 / 0.0555$ & $0.0227 / 0.0586$ & $0.0346 / 0.0738$ \\
\hline
\end{tabular}

a Cu Ka.

b Mo Ka.

c $R 1=\sum\left\|F_{\mathrm{o}}|-| F_{\mathrm{c}}\right\| / \sum F_{\mathrm{o}} \mid w R 2=\left[\sum w\left(\left|F_{\mathrm{o}}\right|-\left|F_{\mathrm{c}}\right|\right)^{2} / \sum w\left|F_{\mathrm{o}}\right|^{2}\right]^{1 / 2}$

\section{Mössbauer Spectroscopy}

The Mössbauer spectra of $\mathrm{Fe}\left[\mathrm{HB}(\mathrm{mtda})_{3}\right]_{2}(4)$ and $\mathrm{Fe}\left[\mathrm{HB}\left(\mathrm{mtda}^{\mathrm{Me}}\right)_{3}\right]_{2}(\mathbf{8})$ have been measured between 4.2 and 295 $\mathrm{K}$ on a constant-acceleration spectrometer that utilized a room temperature rhodium matrix cobalt-57 source and was calibrated at $295 \mathrm{~K}$ with $\alpha$-iron powder. The spectra of 4 and 8 have been measured on absorbers that contained $56 \mathrm{mg} / \mathrm{cm}^{2}$ of sample that had been crushed but not ground and dispersed in boron nitride. Except for the spectra of 4 obtained at 85 and $155 \mathrm{~K}$, which indicate the presence of a small amount of texture, the observed spectra have been fit with symmetric quadrupole doublets. The estimated relative errors are \pm 0.005 $\mathrm{mm} / \mathrm{s}$ for the isomer shifts and quadrupole splittings, $\pm 0.01 \mathrm{~mm} / \mathrm{s}$ for the line widths, and $\pm 0.005(\% \epsilon)(\mathrm{mm} / \mathrm{s})$ for the spectral absorption areas. The absolute errors are approximately twice as large.

\section{Results and Discussion}

\section{Synthesis}

The new alkali metal scorpionates $\mathrm{M}\left[\mathrm{HB}\left(\mathrm{mtda}^{\mathrm{Me}}\right)_{3}\right](\mathrm{M}=\mathrm{Na}(\mathbf{1})$ or $\mathrm{K}(\mathbf{2}))$ are prepared by heating a neat mixture of the appropriate $\mathrm{MBH}_{4}$ with a slight excess (3.5 equiv) of 2-mercapto-5-methyl-1,3,4 thiadiazole, $\mathrm{H}$ (mtdame), to ca. $185^{\circ} \mathrm{C}$ for several hours until 3 equiv of hydrogen is evolved (part a of Scheme 1 ). The use of the slight excess of $\mathrm{H}$ (mtdame) ensures the completion of the 


$$
\begin{aligned}
& \mathrm{M}\left(\mathrm{BH}_{4}\right)+3.5 \mathrm{H}\left(\mathrm{mtda}^{\mathrm{Me}}\right) \frac{\text { neat }}{\Delta, 4 \mathrm{~h}} \longrightarrow \mathrm{M}\left[\mathrm{HB}\left(\mathrm{mtda}^{\mathrm{Me}}\right)_{3}\right]+3 \mathrm{H}_{2}(\mathrm{~g}) \\
& \mathrm{M}=\mathrm{Na} \text { (1) } \\
& M=K(2)
\end{aligned}
$$

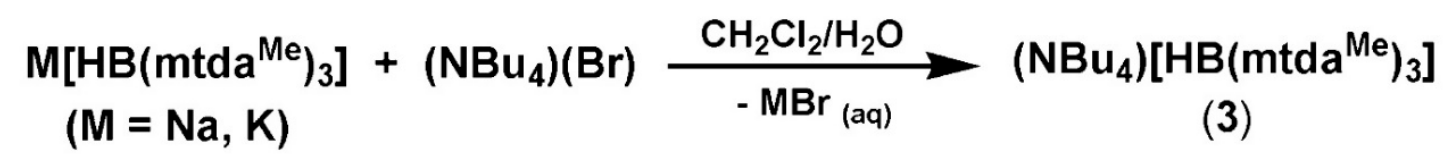

\section{$2\left(\mathrm{NBu}_{4}\right)\left[\mathrm{HB}\left(\mathrm{mtda}^{\mathrm{R}}\right)_{3}\right]+\left[\mathrm{Fe}\left(\mathrm{H}_{2} \mathrm{O}\right)_{6}\right]\left[\mathrm{BF}_{4}\right]_{2}$ $(\mathrm{R}=\mathrm{H}, \mathrm{Me})$

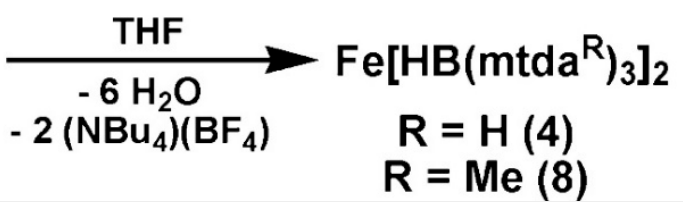

Scheme 1. Syntheses of New Janus Scorpionate Reagents and of Iron(II) Complexes

Reaction, otherwise the product mixture is contaminated with variable amounts of the disubstituted compound $\mathrm{M}\left[\mathrm{H}_{2} \mathrm{~B} \text { (mtdaMe) }\right)_{2}$ (from NMR and ESI(-) MS analysis of the crude mixture), which cannot be successfully separated from the desired compound. Presumably for steric reasons, it has not yet been possible to prepare the tetrasubstituted derivative $\mathrm{M}\left[\mathrm{B}\left(\mathrm{mtda}^{\mathrm{Me}}\right)_{4}\right]$ regardless of the excess heterocycle or reaction temperature. This result is fortuitous in this case as the excess $\mathrm{H}\left(\mathrm{mtda}^{\mathrm{M}}\right)$ ) is easily removed from the desired product by washing with THF. The salts are soluble in $\mathrm{H}_{2} \mathrm{O}$, DMSO, DMF, and methanol, slightly soluble in $\mathrm{CH}_{3} \mathrm{CN}$ and acetone, very slightly soluble in THF, and insoluble in diethyl ether, chlorinated solvents, and hydrocarbons. Both $\mathbf{1}$ and $\mathbf{2}$ are hygroscopic and will tenaciously bind most Lewis basic solvents (vide infra) except for $\mathrm{THF}$ and $\mathrm{Et}_{2} \mathrm{O}$, which can be removed on prolonged heating under vacuum; as such, these compounds are best stored in a drybox. A more convenient reagent for the present work $\left(\mathrm{NBu}_{4}\right)\left[\mathrm{HB}\left(\mathrm{mtda}^{\mathrm{Me}}\right)_{3}\right](3)$ was prepared by the metathetical reaction between $\left(\mathrm{NBu}_{4}\right) \mathrm{Br}$ and either $\mathbf{1}$ or $\mathbf{2}$ in a biphasic mixture of $\mathrm{CH}_{2} \mathrm{Cl}_{2}$ and $\mathrm{H}_{2} \mathrm{O}$ (part $\mathrm{B}$ of Scheme 1 ). The desired $\mathbf{3}$ is freely soluble in solvents such as $\mathrm{CHCl}_{3}, \mathrm{CH}_{2} \mathrm{Cl}_{2}, \mathrm{THF}, \mathrm{CH}_{3} \mathrm{CN}$, and acetone but is not hygroscopic. The iron compounds $\mathrm{Fe}\left[\mathrm{HB}\left(\mathrm{mtda}^{\mathrm{R}}\right)_{3}\right]_{2}(\mathrm{R}=\mathrm{H}(\mathbf{4})$ or $\mathrm{Me}(\mathbf{8}))$ are readily prepared by the reaction between $\left(\mathrm{NBu}_{4}\right)\left[\mathrm{HB}\left(\mathrm{mtda}^{\mathrm{R}}\right)_{3}\right](\mathrm{R}=\mathrm{H}, \mathrm{Me})$ and $\left[\mathrm{Fe}\left(\mathrm{H}_{2} \mathrm{O}\right)_{6}\right]\left(\mathrm{BF}_{4}\right)_{2}$ in THF (part $\mathrm{C}$ of Scheme 1 ), as the desired iron(II) products are insoluble but all of the other species are soluble. The desired iron species $\mathbf{4}$ or $\mathbf{8}$ can also be prepared using $\mathrm{M}\left[\mathrm{HB}\left(\mathrm{mtda}^{\mathrm{R}}\right)_{3}\right](\mathrm{M}=\mathrm{Na}, \mathrm{K} ; \mathrm{R}=\mathrm{H}, \mathrm{Me})$ salts and $\left[\mathrm{Fe}\left(\mathrm{H}_{2} \mathrm{O}\right)_{6}\right]\left(\mathrm{BF}_{4}\right)_{2}$ in $\mathrm{CH}_{3} \mathrm{CN}$; however, the low solubility of the alkali metal reagents (especially for sodium) in this solvent can be problematic. The compound $\mathrm{Fe}\left[\mathrm{HB}(\mathrm{mtda})_{3}\right]_{2}, 4$, is a pink, diamagnetic (low-spin Fe(II)) solid, whereas Fe $\left[\mathrm{HB}\left(\mathrm{mtdame}_{3}\right]_{2}, 8\right.$, is a bright-yellow, paramagnetic $\left[\mu_{\text {eff }}=5.1\right.$ $\mu_{B}$, high-spin Fe(II)] solid. Neither 4 nor 8 exhibit reversible thermochromic behavior as solids that would be indicative of a spin transition. Both $\mathbf{4}$ and $\mathbf{8}$ are insoluble in solvents with which they do not interact. Surprisingly, the compounds react with polar Lewis basic solvents to displace the anionic ligand to varying extents. That is, solid pink $\mathrm{Fe}\left[\mathrm{HB}(\mathrm{mtda})_{3}\right]_{2}$ temporarily gives a pink solution on interaction with $\mathrm{CH}_{3} \mathrm{CN}$ or DMF but the solution quickly $(<1 \mathrm{~s})$ turns pale yellow to colorless. With DMSO or water, the solution immediately becomes colorless with no indication of an initial pink solution. Slow evaporation of water or layering DMSO solution on $\mathrm{CHCl}_{3}$ followed by slow diffusion allowed the isolation crystalline $\left[\mathrm{Fe}\left(\mathrm{H}_{2} \mathrm{O}\right)_{6}\right]\left[\mathrm{HB}(\mathrm{mtda})_{3}\right]_{2} \cdot 6 \mathrm{H}_{2} \mathrm{O}$ $\left(6 \cdot 6 \mathrm{H}_{2} \mathrm{O}\right),\left[\mathrm{Fe}(\mathrm{DMSO})_{6}\right]\left[\mathrm{HB}(\mathrm{mtda})_{3}\right]_{2} \cdot 2 \mathrm{CHCl}_{3}\left(7 \cdot 2 \mathrm{CHCl}_{3}\right)$. Similarly, the bright-yellow $\mathrm{Fe}\left[\mathrm{HB}\left(\mathrm{mtda} \mathrm{Me}_{3}\right]_{2}\right.$ immediately gives colorless to pale-yellow solutions on dissolution in polar solvents $\mathrm{CH}_{3} \mathrm{CN}$, DMF, DMSO, or water. When DMF was used and this solvent was allowed to slowly evaporate, both $\mathrm{Fe}(\mathrm{DMF})_{4}\left[\mathrm{HB}\left(\mathrm{mtdaMe}_{3}\right]_{2}(\mathbf{9})\right.$, and $\left[\mathrm{Fe}(\mathrm{DMF})_{6}\right]\left[\mathrm{HB}\left(\mathrm{mtda}^{\mathrm{Me}}\right)_{3}\right]_{2} \cdot 2 \mathrm{DMF}(\mathbf{1 0} \cdot 2 \mathrm{DMF})$ could be isolated as single crystals, but control over the amount of 
each appears capricious. It should be noted that when the preparation of $\mathbf{8}$ was attempted under aqueous conditions (using either $\mathbf{1}$ or $\mathbf{2}$ and $\mathrm{FeCl}_{2}$ ) the initial product is a colorless solid presumably the hexaaquairon(II) species, which on heating under vacuum gives a yellow tetraaquairon(II) species that only very reluctantly loses water on prolonged heating under vacuum. The water is more rapidly displaced by addition of a weak Lewis base $\left(\mathrm{CH}_{3} \mathrm{CN}\right.$ or THF), filtration, followed by heating under vacuum.

\section{Solid-State Structures}

The structures of the discrete sodium complex $\mathrm{Na}\left[\mathrm{HB}\left(\mathrm{mtda}^{\mathrm{Me}}\right)_{3}\right] \cdot 3(\mathrm{MeOH}), \mathbf{1} \cdot 3 \mathrm{MeOH}$, polymeric $\left\{\mathrm{K}\left[\mathrm{HB}(\mathrm{mtda})_{3}\right] \cdot \mathrm{MeOH}\right\}_{n}$ and $\left\{\mathrm{K}_{2}\left[\mathrm{HB}\left(\mathrm{mtda}^{\mathrm{Me}}\right)_{3}\right]_{2} \cdot 3 \mathrm{MeOH}\right\}_{n}(2 \cdot 1.5 \mathrm{MeOH})$ were obtained and are provided along with detailed discussion in the Supporting Information. The pink iron complex $\mathrm{Fe}\left[\mathrm{HB}(\mathrm{mtda})_{3}\right]_{2}$ crystallizes as tetrasolvates ( $D M F$ or $\mathrm{CH}_{3} \mathrm{CN}$ ) from diethyl ether vapor diffusion into colorless $\mathrm{DMF}$ or $\mathrm{CH}_{3} \mathrm{CN}$ solutions, as appropriate. The structure of the solvent-free complex has not yet been possible to obtain. Also, the solventfree (or any noncoordinated solvate of) the bright yellow $\mathrm{Fe}\left[\mathrm{HB}\left(\mathrm{mtda}^{\mathrm{Me}}\right)_{3}\right]_{2}$ has defied all attempts at crystallization. Figure $\underline{4}$ shows the molecular structure of $\mathrm{Fe}\left[\mathrm{HB}(\mathrm{mtda})_{3}\right]_{2} \cdot 4 \mathrm{DMF}(4 \cdot 4 \mathrm{DMF})$, whereas that of the isostructural tetra-acetonitrile solvate $4 \cdot 4 \mathrm{CH}_{3} \mathrm{CN}$ is included in the Supporting Information. The structure of 4.4DMF has two scorpionate ligands each bound in a $\mathrm{K}^{3}$ manner to iron(II) via nitrogen donors to give a pseudooctahedral FeN $\mathrm{N}_{6}$ kernel; the molecules of solvation are not bound to the metal center. The average Fe-N distance of $1.97 \AA$ is characteristic for low-spin iron(II) being slightly shorter than the average distance of $1.98 \AA$ found in $\mathrm{Fe}\left[\mathrm{HB}(\mathrm{pz})_{3}\right]_{2},(2 \mathrm{C})$ which is also low spin iron(II) at or below room temperature.(23) Of interest, is the degree of ligand distortion in $\mathrm{k}^{3} \mathrm{~N}$-coordinated scorpionate ligands, which is commonly manifested in any of three ways: $\mathrm{M}-\mathrm{N}$ bond lengthening, heterocycle ring twisting, and boron pyramidalization, as summarized in Figure $\underline{5}$. The $\mathrm{M}-\mathrm{N}$ bond lengthening distortion is easy to measure and the effects are obvious. The two other distortions, ring-twisting and boron pyramidalization require more sophisticated measurements and their effects are more subtle than metal-ligand bond lengthening. Ring twisting is measured by the MN-NB torsion angle; a metal-bonded scorpionate fragment with ideal $C_{3 v}$ symmetry is expected to have a MN-NB torsion angle of $0^{\circ}$. For a given scorpionate with a generally fixed bite angle, the degree of ring twisting increases with the size of the metal. For distortions caused by boron pyramidalization (shown in the right of Figure $\underline{5}$ ), the B-N bond distances and $\mathrm{B}-\mathrm{N}-\mathrm{N}$ angles remain fixed but the $\mathrm{N}-\mathrm{B}-\mathrm{N}$ angles change. This distortion can be measured by the distance between boron and the centroid of the boron-bound nitrogens, $\mathrm{B}-\mathrm{Ct}\left(\mathrm{N}_{3}\right)$. An ideal $\mathrm{B}-\mathrm{Ct}\left(\mathrm{N}_{3}\right)$ distance of $0.52 \AA \AA$ can be estimated $\left[=(1.55 \AA) \cos \left(180-109.5^{\circ}\right)\right]$ based on the average $B-N$ distance of $1.55 \AA$ found for all the known boron-mercaptothiadiazolyl complexes and by using an ideal tetrahedral N-B-N bond angle. As a matter of perspective, a related study has shown that the $\mathrm{B}-\mathrm{Ct}\left(\mathrm{N}_{3}\right)$ distance varies between 0.48 to $0.62 \AA$ for tris(pyrazolyl)borate complexes of iron(II) in high-spin and low-spin states, where the effective sizes of the metal center in each spin state is strikingly different. $(2,23)$ As with the tris(pyrazolyl)borate, there is very little heterocycle ring-twisting with the average twist angle FeN-NB of $2.33^{\circ}$ for 4.4DMF, which compares favorably to the average of $1.85^{\circ}$ found for $\mathrm{Fe}\left[\mathrm{HB}(\mathrm{pz})_{3}\right]_{2}$. (23) The relatively large $\mathrm{B}-\mathrm{Ct}\left(\mathrm{N}_{3}\right)$ distance in $4 \cdot 4 \mathrm{DMF}$ of $0.57 \AA$ likely reflects the shorter average Fe-N bond distance and can be compared to the average value of $0.56 \AA$ found in $\mathrm{Fe}\left[\mathrm{HB}(\mathrm{pz})_{3}\right]_{2}$. 

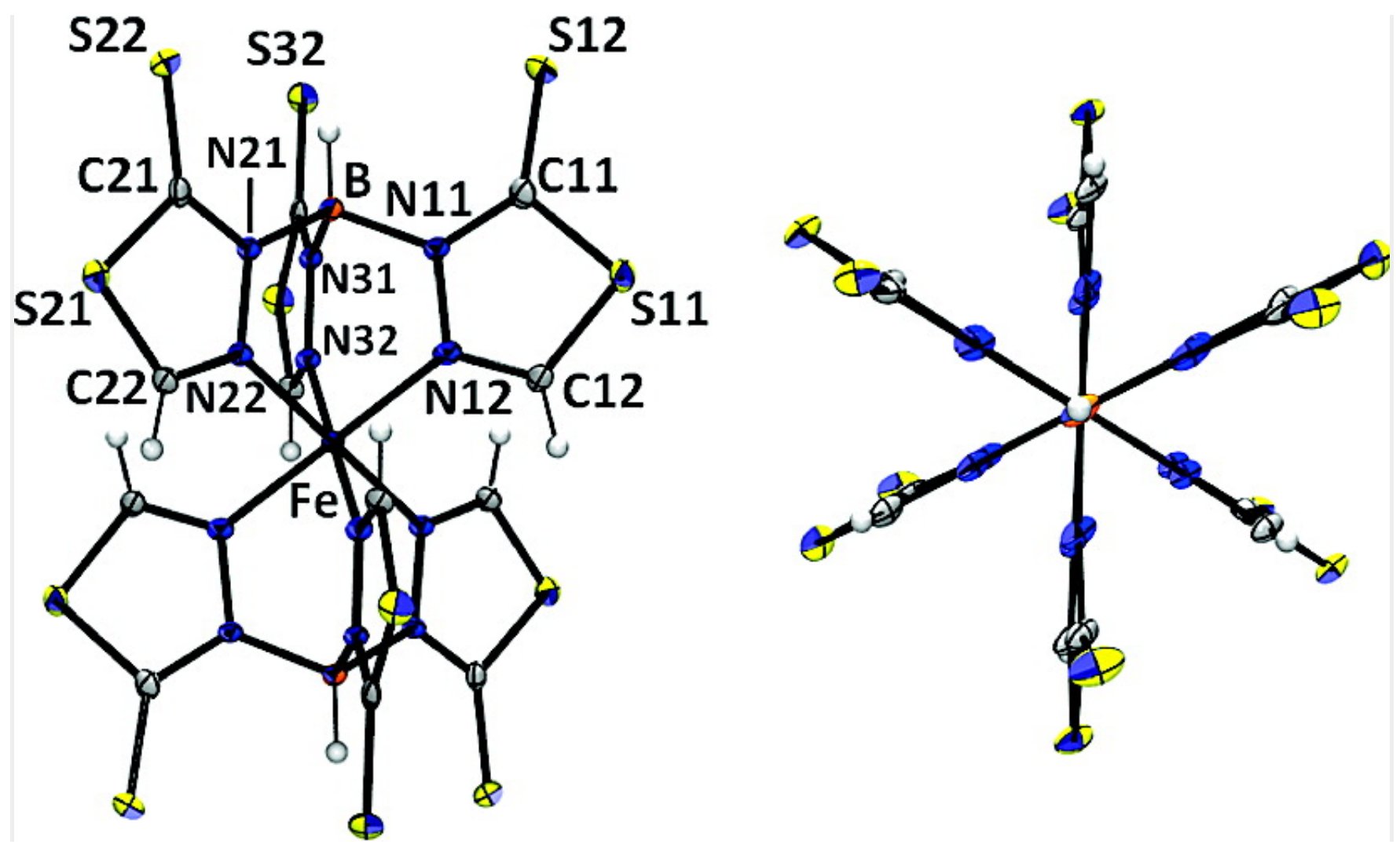

Figure 4. Crystal structure of $\mathrm{Fe}\left[\mathrm{HB}(\mathrm{mtda})_{3}\right]_{2} \cdot 4 \mathrm{DMF}$, 4.4DMF, (ORTEP displacement ellipsoids shown at $50 \%$ probability; DMF molecules removed for clarity) with a view down 3-fold axis.

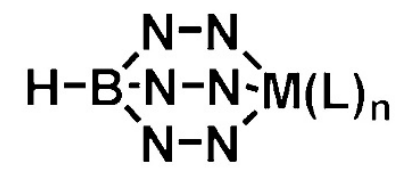

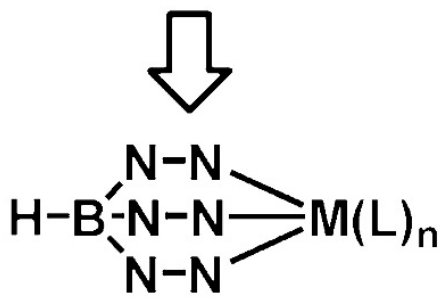

M-N bond-lengthening<smiles>B1NNNNN1</smiles>

ring twisting<smiles></smiles>

boron pyramidalization

Figure 5. Common distortions in metal scorpionates where $\mathrm{N}-\mathrm{N}$ represents diazolyl ring such as a thiadiazolyl or even a pyrazolyl.

As described above, slow evaporation of DMF or $\mathrm{H}_{2} \mathrm{O}$ solutions of $\mathrm{Fe}\left[\mathrm{HB}\left(\mathrm{mtda}^{\mathrm{R}}\right)_{3}\right]_{2}(\mathrm{R}=\mathrm{H}, \mathrm{Me})$ or slow diffusion of $\mathrm{CHCl}_{3}$ into a DMSO solution of $\mathrm{Fe}\left[\mathrm{HB}\left(\mathrm{mtda}^{\mathrm{Me}}\right)_{3}\right]_{2}$ permitted the isolation of single crystals of hexa(solvent)iron complexes where the solvent either partially or completely displaces the anionic tripodal ligands.

Figure $\underline{6}$ provides the crystal structures of $\left[\mathrm{Fe}\left(\mathrm{H}_{2} \mathrm{O}\right)_{6}\right]\left[\mathrm{HB}(\mathrm{mtda})_{3}\right]_{2} \cdot 6 \mathrm{H}_{2} \mathrm{O}\left(6 \cdot 6 \mathrm{H}_{2} \mathrm{O}\right)$,

$\left[\mathrm{Fe}(\mathrm{DMSO})_{6}\right]\left[\mathrm{HB}(\mathrm{mtda})_{3}\right]_{2} \cdot 2 \mathrm{CHCl}_{3}\left(\mathbf{7} \cdot 2 \mathrm{CHCl}_{3}\right), \mathrm{Fe}(\mathrm{DMF})_{4}\left[\mathrm{HB}\left(\mathrm{mtda}^{\mathrm{Me}}\right)_{3}\right]_{2}(\mathbf{9})$, and $\left[\mathrm{Fe}(\mathrm{DMF})_{6}\right]\left[\mathrm{HB}\left(\mathrm{mtda} \mathrm{Me}_{3}\right]_{2} \cdot 2 \mathrm{DMF}\right.$ 
(10.2DMF). In all cases, iron is located at an inversion center rendering the two scorpionate ligands equivalent by symmetry. The scorpionate ligands maintain a conformation where all sulfurs are directed toward the boron-hydride group. In $6 \cdot 6 \mathrm{H}_{2} \mathrm{O}, 7 \cdot 2 \mathrm{CHCl}_{3}$, and $\mathbf{1 0} \cdot 2 \mathrm{DMF}$, the iron compounds exist as ion pairs with [Fe(solvent) $\left.]_{6}\right]^{2+}$ cations (solvent $=\mathrm{H}_{2} \mathrm{O}, \mathrm{DMSO}$, or $\mathrm{DMF}$ ) with $\mathrm{FeO}_{6}$ coordination spheres and free Janus scorpionate anions $\left[\mathrm{HB}\left(\mathrm{mtda}^{\mathrm{R}}\right)_{3}\right]^{-}(\mathrm{R}=\mathrm{H}$ or $\mathrm{Me})$, as appropriate. The average $\mathrm{Fe}-\mathrm{O}$ distances in the dications are comparable to related, previously reported high-spin iron(II) complexes: $2.10 \AA$ for $6 \cdot 6 \mathrm{H}_{2} \mathrm{O}$ (compare to avg. $\mathrm{Fe}-\mathrm{O}$ of $2.11 \AA$ for $\left.\left[\mathrm{Fe}^{\prime \prime}\left(\mathrm{H}_{2} \mathrm{O}\right)_{6}\right]\left(\mathrm{CF}_{3} \mathrm{SO}_{3}\right)_{2}(24)\right), 2.13 \AA$ for $7 \cdot 2 \mathrm{CHCl}_{3}$ (compare to avg. Fe-O of $2.13 \AA$ for [Fe"(DMSO) $\left.)_{6}\right]\left(\mathrm{Cl}_{2} \mathrm{FeMoS}_{4}\right)(25)$ ), and $2.12 \AA$ for $10 \cdot 2 \mathrm{DMF}$ (compare to avg. Fe-O of $2.12 \AA$ for $\left.\left[\mathrm{Fe} "(\mathrm{DMF})_{6}\right]\left(\mathrm{Cl}_{2} \mathrm{FeS}_{4} \mathrm{~W}\right)(25)\right)$. With $\mathbf{6} \cdot 6 \mathrm{H}_{2} \mathrm{O}$, there is an extensive hydrogen bonding network found in the solid state that involves the anionic scorpionate ligand, (Supporting Information). Most striking is the contrast in the connectivity between the structures of the two complexes with the formula $\mathrm{Fe}\left[\mathrm{HB}\left(\mathrm{mtda}^{\mathrm{R}}\right)_{3}\right]_{2} \cdot 4 \mathrm{DMF}(\mathrm{R}=\mathrm{H}, \mathrm{Me})$. The structure of the first-generation $(R=H)$ ligand showed two facially coordinating ligands and no metal bound solvent molecules (vide supra), whereas that of the second-generation ligand has two trans-K ${ }^{1} \mathrm{~S}$ - coordinated scorpionates and four iron-bound DMF molecules. Both the average Fe-O distance of $2.12 \AA$ and average Fe-S distance of $2.51 \AA$ are characteristic for high-spin iron(II). The former distance is identical to that found in 10.2DMF and [ $\left.\mathrm{Fell}^{\prime \prime}(\mathrm{DMF})_{6}\right]\left(\mathrm{Cl}_{2} \mathrm{FeS}_{4} \mathrm{~W}\right)$, whereas the latter compares favorably with other high-spin iron(II) complexes of thione donors such as $2.46 \AA$ in $\mathrm{Fe}(\mathrm{Tm})_{2} \cdot 4.5 \mathrm{H}_{2} \mathrm{O} \underline{(13)}$ and $2.57 \AA$ for

$\left[\mathrm{Fe}(\mathrm{DMTU})_{6}\right]\left(\mathrm{BF}_{4}\right)_{2} \cdot \mathrm{DMTU}(26)$ (DMTU= N,N'-dimethylthiourea). Low-spin iron(II) complexes of sulfur donors typically have Fe-S distances closer to $2.30 \AA$, such as found in Riordan's Fe(RTt) ${ }_{2} \operatorname{complexes}(27)(\mathrm{R}=$ (methylthio)methyl or phenyl; Tt - tris(methylthio)methylborate) with avg. Fe-S of $2.303 \AA$, or in [Fe(1,4,7trithiacyclononane $\left.)_{2}\right]\left(\mathrm{PF}_{6}\right)_{2}(28)$ with an average $\mathrm{Fe}-\mathrm{S}$ distance of $2.250 \AA$, as representative examples. 

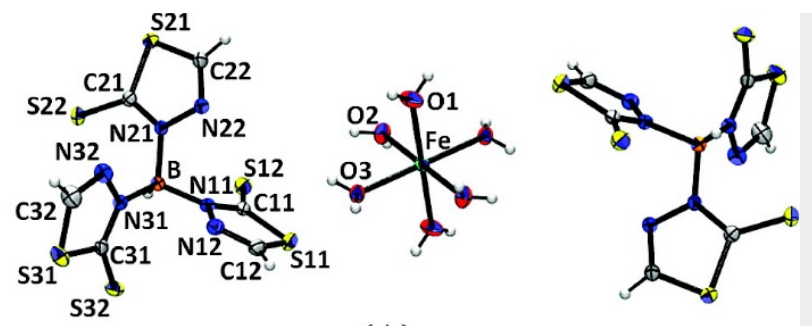

(A)

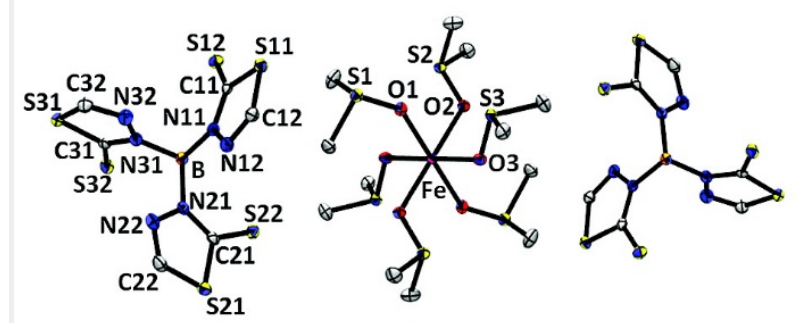

(B)

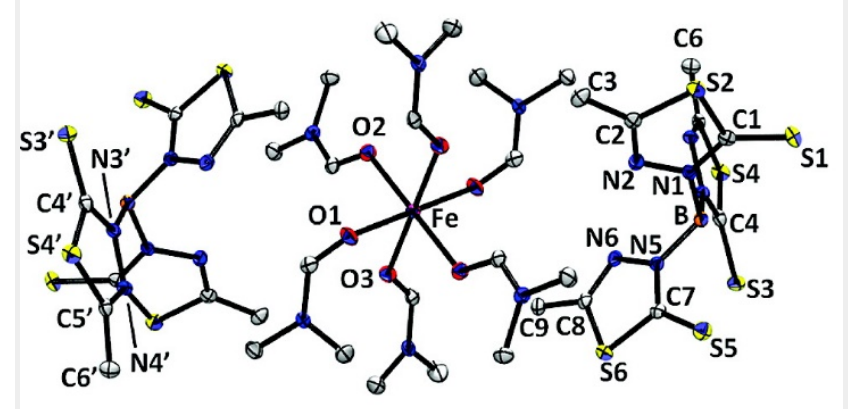

(C)

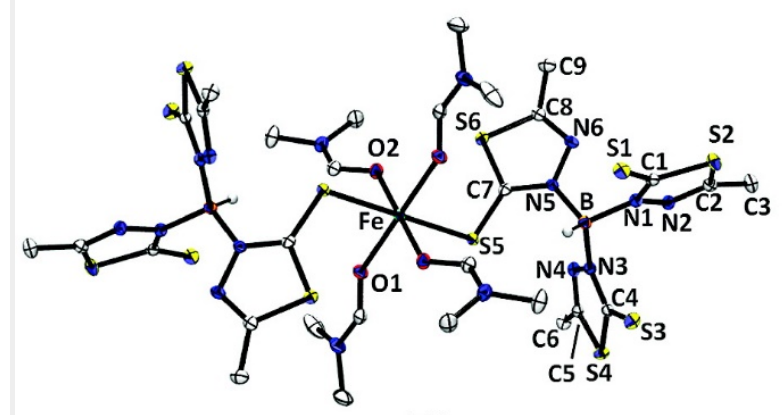

(D)

Figure 6. ORTEP diagrams (50\% probability ellipsoids) of $(\mathrm{A})\left[\mathrm{Fe}\left(\mathrm{H}_{2} \mathrm{O}\right)_{6}\right]\left[\mathrm{HB}(\mathrm{mtda})_{3}\right]_{2} \cdot 6 \mathrm{H}_{2} \mathrm{O}, 6 \cdot 6 \mathrm{H}_{2} \mathrm{O}$, (B) $\left[\mathrm{Fe}(\mathrm{DMSO})_{6}\right]\left[\mathrm{HB}(\mathrm{mtda})_{3}\right]_{2} \cdot 2 \mathrm{CHCl}_{3}, 7 \cdot 2 \mathrm{CHCl}_{3},(\mathrm{C})\left[\mathrm{Fe}(\mathrm{DMF})_{6}\right]\left[\mathrm{HB}(\mathrm{mtdame})_{3}\right]_{2} \cdot 2 \mathrm{DMF}, 10 \cdot 2 \mathrm{DMF}$, and (D) Fe(DMF) ${ }_{4}\left[\mathrm{~K}^{1} \mathrm{~S}-\right.$ $\mathrm{HB}\left(\mathrm{mtdame}_{3}\right]_{2}, 9$, with most hydrogens and all noncoordinated solvents removed for clarity.

\section{Mössbauer spectra}

To probe the nature of ligand binding in solvent-free $\mathrm{Fe}\left[\mathrm{HB}\left(\mathrm{mtda}^{\mathrm{R}}\right)_{3}\right]_{2}(\mathrm{R}=\mathrm{H}(\mathbf{4}), \mathrm{Me}(\mathbf{8}))$, for which solid-state structural data could not be obtained, the Mössbauer spectra have been measured and the spectral results have been compared with each other and with related complexes. The Mössbauer spectra of $\mathbf{4}$ and $\mathbf{8}$, obtained between 4.2 and $295 \mathrm{~K}$, have been fit with a single quadrupole doublet with a single line width but with slightly 
different areas for the two components of the spectra of 4 at 85 and $155 \mathrm{~K}$; the resulting parameters are given in Table 3 , and the $4.2,85$, and $295 \mathrm{~K}$ spectra of 4 and $\mathbf{8}$ are shown in the left and right of Figure $\underline{7}$, respectively. The remaining spectra measured at other temperatures are all very similar to those shown in these figures. High-temperature spectra of $\mathbf{4}$ were not pursued, as there was no indication of spin-transition behavior (color change) before thermal decomposition.

Table 3. Mössbauer Spectral Parameters

\begin{tabular}{|c|c|c|c|c|c|}
\hline compound & $T, \mathrm{~K}$ & $\delta, \mathrm{mm} / \mathrm{s}^{\mathrm{a}}$ & $\Delta E_{Q}, \mathrm{~mm} / \mathrm{s}$ & $\Gamma, \mathrm{mm} / \mathrm{s}$ & area, $(\% \in)(\mathrm{mm} / \mathrm{s})$ \\
\hline \multirow[t]{7}{*}{$\mathrm{Fe}\left[\mathrm{HB}(\mathrm{mtda})_{3}\right]_{2}, \mathbf{8}$} & 295 & 0.408 & 0.272 & 0.29 & 2.524 \\
\hline & 225 & 0.440 & 0.282 & 0.29 & 3.474 \\
\hline & 155 & 0.469 & 0.278 & 0.30 & 4.628 \\
\hline & 85 & 0.481 & 0.280 & 0.29 & 5.685 \\
\hline & 55 & 0.487 & 0.283 & 0.35 & 6.622 \\
\hline & 25 & 0.490 & 0.276 & 0.33 & 6.960 \\
\hline & 4.2 & 0.489 & 0.264 & 0.31 & 7.179 \\
\hline \multirow[t]{7}{*}{$\mathrm{Fe}\left[\mathrm{HB}\left(\mathrm{mtda}^{\mathrm{Me}}\right)_{3}\right]_{2}, \mathbf{8}$} & 295 & 0.847 & 1.442 & 0.34 & 1.202 \\
\hline & 225 & 0.898 & 1.678 & 0.35 & 1.944 \\
\hline & 155 & 0.942 & 1.990 & 0.37 & 3.274 \\
\hline & 85 & 0.976 & 2.228 & 0.36 & 4.730 \\
\hline & 55 & 0.988 & 2.314 & 0.36 & 5.251 \\
\hline & 25 & 0.990 & 2.316 & 0.36 & 5.890 \\
\hline & 4.2 & 0.988 & 2.299 & 0.34 & 6.366 \\
\hline
\end{tabular}

a The isomer shifts are given relative to room temperature $\alpha$-iron powder. 


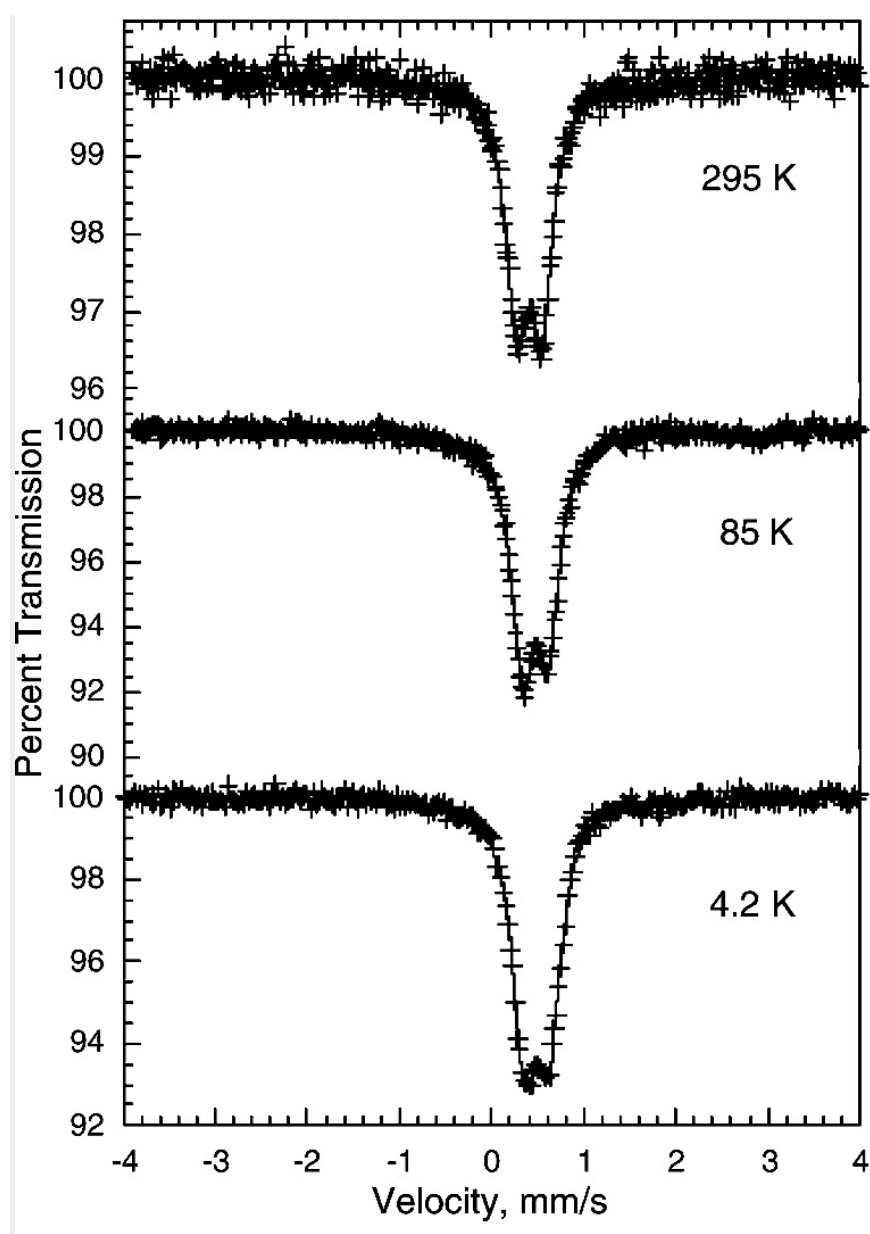

$\mathrm{Fe}\left[\mathrm{HB}(\mathrm{mtda})_{3}\right]_{2}, 4$

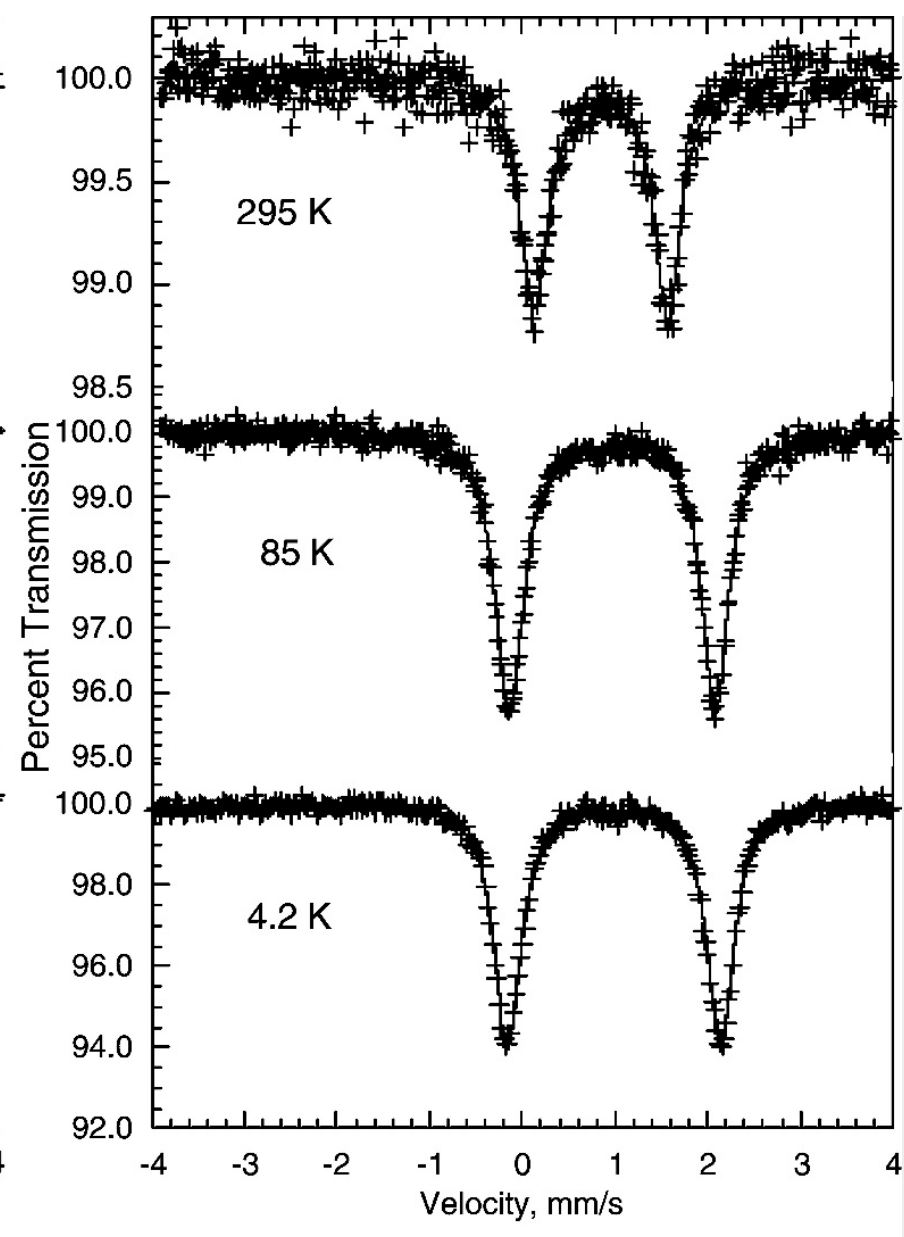

$\mathrm{Fe}\left[\mathrm{HB}\left(\mathrm{mtda}^{\mathrm{Me}}\right)_{3}\right]_{2}, 8$

Figure 7. The iron-57 Mössbauer spectra of $\mathrm{Fe}\left[\mathrm{HB}(\mathrm{mtda})_{3}\right]_{2}, 4$, (left) and of $\mathrm{Fe}\left[\mathrm{HB}(\text { mtdame })_{3}\right]_{2}, 8$, (right), obtained at $4.2,85$, and $295 \mathrm{~K}$.

The Mössbauer spectra and the derived hyperfine parameters of $\mathrm{Fe}\left[\mathrm{HB}(\mathrm{mtda})_{3}\right]_{2}, 4$, are fully consistent $(23$, 29) with the presence of low-spin iron(II) in a slightly distorted pyrazolylborate-type $\mathrm{FeN}_{6} \mathrm{C}_{\text {coordination }}$ environment; there is no indication of the presence of any high-spin iron(II). The temperature dependence of the isomer shift, $\delta$, of 4 (Figure 8) is well fit with the Debye model(30)for the second-order Doppler shift with a characteristic Mössbauer temperature, $\Theta_{M}{ }^{\delta}$, of $690(20) \mathrm{K}$. This temperature is much larger than the Mössbauer temperature, $\Theta_{M}{ }^{A}$, of $183(2) \mathrm{K}$ obtained from the temperature dependence of the logarithm of the spectral absorption area (Figure 8). It is well-known(31-33) that the two Mössbauer temperatures, $\Theta_{M}{ }^{\delta}$ and $\Theta_{M^{A}}$, obtained from the two temperature dependencies are different $(33)$ because they depend, for the isomer shift, on $\left\langle v^{2}\right\rangle$, the mean-square vibrational velocity of the iron-57, a velocity that is mostly dependent upon optical molecular vibrations in the energy range of ca. 50 to $100 \mathrm{meV}$ or 400 to $800 \mathrm{~cm}^{-1}$ and, for the absorption area, on $\left\langle x^{2}\right\rangle$, the mean-square displacement of the iron-57, a displacement that is strongly affected by acoustical lattice vibrations at ca. $30 \mathrm{meV}$ or $250 \mathrm{~cm}^{-1}$. Measurements of the Mössbauer temperatures of related iron(II) complexes $(24,30,32)$ and iron nitroprussides(33)indicate that $\Theta_{M}{ }^{8}$ is twice to five times $\Theta_{M}{ }^{A}$. The present factor of ca. 4 is in this range. The isomer shift of 4 (Table $\underline{3}$ and Figure $\underline{8}$ ) decreases by only $0.081 \mathrm{~mm} / \mathrm{s}$ between 4.2 and 
$295 \mathrm{~K}$, or far less than would be expected on the basis of the 183(2) K value of $\Theta_{M^{A}}$. Further, the larger $\Theta_{M}{ }^{\delta}$ observed in low-spin 4 as compared to high-spin $\mathbf{8}$ (below) is consistent with the higher-energy vibrational frequencies at ca. $400 \mathrm{~cm}^{-1}$ observed in low-spin iron(II) complexes as compared to lower-energy vibrational frequencies found at ca. $250 \mathrm{~cm}^{-1}$ in high-spin iron(II) complexes.(34)

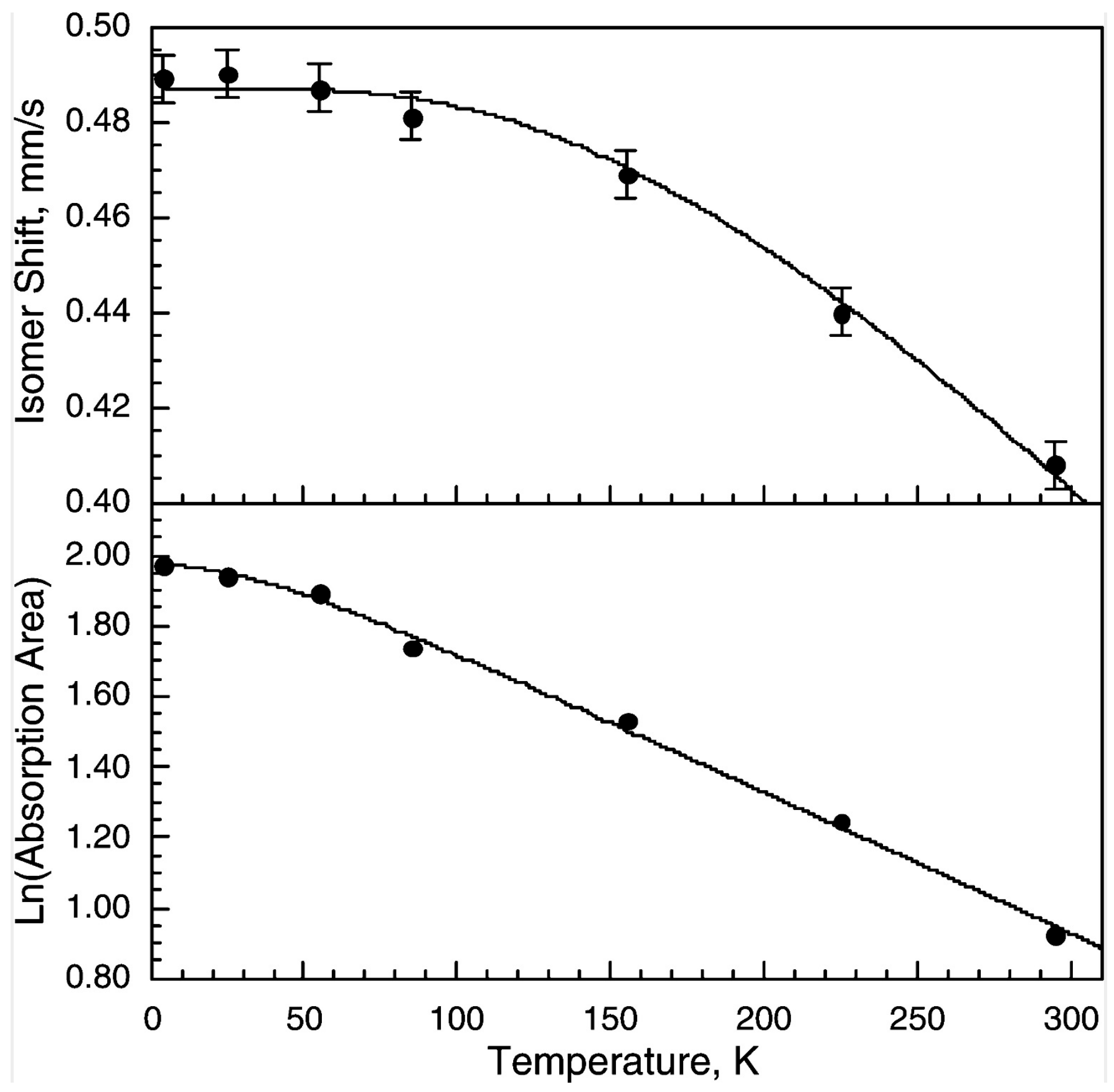

Figure 8. The temperature dependence of the isomer shift, upper plot, and the logarithm of the spectral absorption area, lower plot, of $\mathrm{Fe}\left[\mathrm{HB}(\mathrm{mtda})_{3}\right]_{2}, 4$, and fit with the Debye model. 
The Mössbauer spectral hyperfine parameters of $\mathrm{Fe}\left[\mathrm{HB}\left(\mathrm{mtdame}_{3}\right]_{2}, \mathbf{8}\right.$, are very different from those of $\mathrm{Fe}\left[\mathrm{HB}(\mathrm{mtda})_{3}\right]_{2}, 4$. In this case, because of the complex nature of the Janus scorpionate ligands the iron(II) coordination environment could consist of $\mathrm{FeN}_{6}, \mathrm{FeS}_{6}$, or $\mathrm{FeN}_{3} \mathrm{~S}_{3}$, or, perhaps less likely, some combination of these environments. The observed isomer shift of $0.847 \mathrm{~mm} / \mathrm{s}$ at $295 \mathrm{~K}$ is clearly smaller than is typically observed for high-spin iron(II) in the $\mathrm{FeN}_{6}$ coordination of pyrazolylborate ligands, $(2 \mathrm{~d}, 23,29,32)$ but this isomer shift is fully consistent with the $\mathrm{FeS}_{6}$ coordination environment, in which the soft-base coordinated sulfur leads to a higher s-electron density at the iron-57 nucleus and, consequently, a lower isomer shift. In addition, the temperature dependence of the isomer shift of 8 yields a $\Theta_{M}{ }^{\delta}$ temperature of $318(8) \mathrm{K}$ (Figure $\underline{9}$ ) a value that is typical of the weaker Fe-S bonding than the alternative Fe- $\mathrm{N}$ bonding. A fit of the logarithm of the spectral absorption area (Figure 9 ) yields a $\Theta_{M^{A}}$ temperature of $147(2) \mathrm{K}$, a value significantly lower, as expected for the

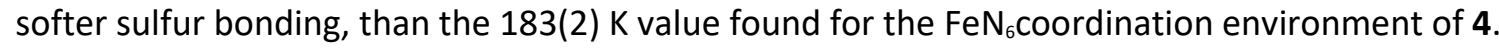

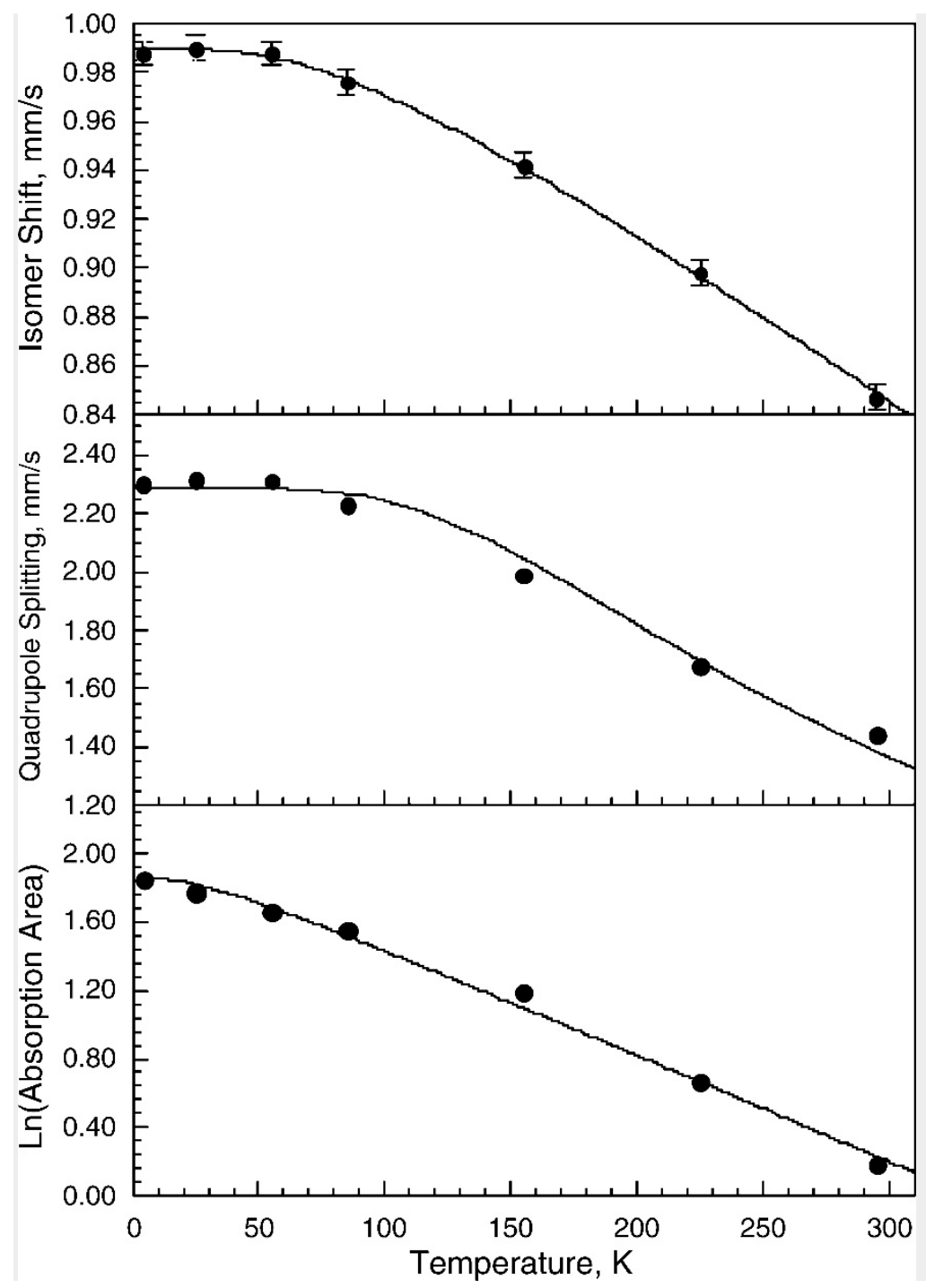

Figure 9. The temperature dependence of the isomer shift, upper, the quadrupole splitting, center, and the logarithm of the spectral absorption area, lower, of $\mathrm{Fe}\left[\mathrm{HB}\left(\mathrm{mtdame}_{3}\right]_{2}, \mathbf{8}\right.$. The isomer shift and the logarithm of the spectral absorption area have been fit with the Debye model and the quadrupole splitting with the Ingalls model. 
It is more difficult to determine whether some, or perhaps all, of the iron(II) in $\mathbf{8}$ has the $\mathrm{FeN}_{3} \mathrm{~S}_{3}$, coordination environment or some mixture of the possibilities mentioned above. The observed spectra are clean and well resolved and offer no indication of more than a unique iron(II) site. However, it should be noted that the line widths of ca. 0.34 to $0.37 \mathrm{~mm} / \mathrm{s}$ observed for 8 are substantially larger than the 0.29 to $0.33 \mathrm{~mm} / \mathrm{s}$ values observed for 4 and the values of 0.24 to $0.25 \mathrm{~mm} / \mathrm{s}$ observed for many related complexes; the inner lines of the iron powder calibration are typically 0.24 to $0.25 \mathrm{~mm} / \mathrm{s}$. Thus, the larger than expected line width may be an indication of some disorder in the coordination environment, but there is no other such indication in the Mössbauer spectra of $\mathbf{8}$.

As is always the case for high-spin iron(II) complexes, the quadrupole splitting depends upon two contributions to the electric field gradient, a lattice contribution, $q_{\text {lat }}$ and a valence contribution, $q_{\text {val }}$. The former is usually small and essentially independent of temperature such that the magnitude and temperature dependence of the quadrupole splitting is dominated by the valence contribution. The Ingalls model (35) has been used to fit the

temperature dependence of the iron(II) quadrupole splitting of $\mathbf{8}$, and the results are shown as the line in the center of Figure $\underline{9}$. The fit yields $354(5) \mathrm{cm}^{-1}$ for the splitting of the ground state $t_{2 g}$ orbitals of 8 by a lowsymmetry component of a pseudo-octahedral coordination environment about iron(II).

\section{Conclusions}

The new second-generation Janus scorpionate ligand, $\left[\mathrm{HB}\left(\mathrm{mtda}^{\mathrm{Me}}\right)_{3}\right]^{-}$, has been prepared as either a sodium or potassium complex by heating a neat mixture of the appropriate $\mathrm{MBH}_{4}(\mathrm{M}=\mathrm{Na}$ or $\mathrm{K}$ ) and an excess (>3 equiv) of heterocycle. This new anionic ligand differs from that previously reported due to the presence of methyl groups situated proximal to the nitrogen atoms of the constituent heterocycles that would be available for metal binding. It was expected that steric interactions involving the methyl groups might change the binding preferences of the ligand to metal cations by decreasing the tendency for $\mathrm{MN}_{6}$ coordination in sandwich complexes or coordination polymers, possibly promoting lower coordination numbers or making metal-sulfur bonding more favorable. The results presented here appear consistent with this hypothesis, especially in the case of the iron(II) complexes. The striking contrast in the structures of the tetra-DMF solvates of each $\mathrm{Fe}\left[\mathrm{HB}\left(\mathrm{mtda}^{\mathrm{R}}\right)_{3}\right]_{2}(\mathrm{R}=\mathrm{H}(\mathbf{4}), \mathrm{Me}(\mathbf{8}))$ provides a clear illustration of the distinction between the first-generation ( $\mathrm{R}$ $=\mathrm{H})$ and second-generation $\left(\mathrm{R}=\mathrm{Me}\right.$ ) ligands. In the pink, low-spin Fe $\left[\mathrm{HB}(\mathrm{mtda})_{3}\right]_{2} \cdot 4 \mathrm{DMF}$, both first-generation ligands are bound to iron in a $\mathrm{K}^{3}$-mode and solvent is not, whereas in the yellow high-spin $\mathrm{Fe}\left[\mathrm{HB}(\mathrm{mtdaMe})_{3}\right]_{2} \cdot 4 \mathrm{DMF}$ both second generation scorpionate ligands are bound in a $\mathrm{K}^{1} \mathrm{~S}$ mode trans to each other with four equatorially bound solvent molecules completing the coordination sphere of iron. Whereas the structures of the solvent-free forms of the iron compounds are not known, the discrepancy in binding modes for the different generations of ligand are apparent from Mössbauer and magnetic data. Mössbauer data indicate that the pink diamagnetic parent compound $\mathrm{Fe}\left[\mathrm{HB}(\mathrm{mtda})_{3}\right]_{2} 4$ is low-spin with a $\mathrm{FeN}_{6}$ coordination environment, whereas the paramagnetic methyl-substituted derivative $\mathrm{Fe}\left[\mathrm{HB}\left(\mathrm{mtda}^{\mathrm{me}}\right)_{3}\right]_{2} 8$ is high-spin. In contrast to the parent compound, the iron(II) center of $\mathbf{8}$ is not in an $\mathrm{FeN}_{6}$ environment, rather it is most likely in a $\mathrm{FeS}_{6}$ or possibly a $\mathrm{FeN}_{3} \mathrm{~S}_{3}$ environment. The $\mathrm{FeS}_{6}$ coordination environment is further suggested from the structures of $\left[\mathrm{Fe}(\mathrm{DMF})_{6}\right]\left[\mathrm{HB}\left(\mathrm{mtda}^{\mathrm{Me}}\right)_{3}\right]_{2} \cdot 2 \mathrm{DMF}$ and $\mathrm{Fe}(\mathrm{DMF})_{4}\left[\mathrm{~K}^{1} \mathrm{~S}-\mathrm{HB}\left(\mathrm{mtda}^{\mathrm{Me}}\right)_{3}\right]_{2}$ where each of the two ligands in the former complex was not bound to iron but, in the latter, each was identically bound (in a symmetric, trans fashion) to iron via one sulfur, as a result of displacing coordinated DMF. By extension, it can be envisioned that such substitution continues until no more solvent is bound to the metal. Because the ligand appears to always adopt a conformation where all sulfurs are on the same face, a $\mathrm{FeS}_{6}$ environment can be confidently proposed, although it remains unclear if the ligand binds in a $f a c-\mathrm{K}^{3} \mathrm{~S}_{3}$ manner or in a bridging mode to give a coordination 
polymer. The astonishing range of coordination capabilities that the Janus scorpionate ligand can exhibit (binding from zero to five metals) and the apparent control of binding preferences of this ligand to metal cations by size selection or via steric protection of the nitrogenous face warrant further investigation. Our current endeavors are toward the development and coordination chemistry of second-generation Janus scorpionates with more sterically demanding substituents than methyls.

\section{Acknowledgment}

J.R.G. thanks Marquette University and the Petroleum Research Fund for financial support. F.G. acknowledges with thanks the financial support of the Fonds National de la Recherche Scientifique, Belgium, through Grants 9.456595 and 1.5.064.05.

\section{References}

1. (a) Trofimenko, , S. J. Am. Chem. Soc.1966, 88,, 1842. (b) Trofimenko,, S. Scorpionates: The Coordination Chemistry of Polypyrazolylborate Ligands; 1999

2. (a) Jesson, J. P., Trofimenko, S., and Eaton, D. R. J. Am. Chem. Soc. 1967, 89, 3158 (b) Jesson, J. P., Weiher, J. F., and Trofimenko, S. J. Chem. Phys. 1968, 48,2058 (c) Oliver, J. D., Mullica, D. F., Hutchinson, B. B., and Milligan, W. O.Inorg. Chem. 1980, 19, 165 (d) Long, G. J. and Hutchinson, B. B. Inorg.

Chem. 1987, 26, 608

3. (a) Gorrell, I. B. and Parkin, G. Inorg. Chem. 1990, 29, 2452 (b)Chen, J. and Woo, L. K. J. Organomet. Chem. 2000, 601, 57

4. Remacle,, F.; , Grandjean, F.;, and Long, G. J. Inorg. Chem. 2008, 47, 4005.

5. Long, G. J., Grandjean, F., and Reger, D. L. Spin Crossover in Pyrazolylborate and Pyrazolylmethane Complexes, In Spin Crossover in Transition Metal Compounds I; Gütlich, P. and Goodwin, H. A., Eds.,Springer: Berlin, 2004; 233, 91- 122.

6. (a) Betley, T. A. and Peters, J. C. Inorg. Chem. 2003, 42, 5074 (b)Shapiro, I. R., Jenkins, D. M., Thomas, J. C., Day, M. W., and Peters, J. C. Chem. Commun. 2001,2152

7. (a) Patel, D. V., Mihalcik, D. J., Kreisel, K. A., Yap, G. P. A., Zakharov, L. N., Kassel, W. S., Rheingold, A. L., and Rabinovich, D. Dalton Trans. 2005, 2410 (b) Patel, D. V.,Kriesel, K. A., Yap, G. P. A., and Rabinovich, D. Inorg. Chem. Commun. 2006, 9, 748

8. (a) Fujita, K., Rheingold, A. L., and Riordan, C. G. Dalton Trans. 2003, 2004 (b)Schebler, P. J., Riordan, C. G., Guzei, I. A., and Rheingold, A. L. Inorg. Chem. 1998, 37, 4754

9. Kimblin, C., Bridgewater, B. M., Hascall, T., and Parkin, G. J. Chem. Soc., Dalton Trans. 2000,1267

10. Cetin, A and Ziegler, C. Dalton Trans. 2006, 1006

11. Bao, M., Hayashi, T., and Shimada, S. Dalton Trans. 2004, 2055

12. (a) Ojo, J. F., Slavin, P. A., Reglinski, J., Garner, M., Spicer, M. D., Kennedy, A. R., and Treat, S. J. Inorg. Chim. Acta 2001, 313, 15 (b) Garner, M., Reglinski, J., Cassidy, I.,Spicer, M. D., and Kennedy, A. R. J. Chem. Soc., Chem. Commun. 1996, 1975

13. Garner, M., Lewinski, K., Pattek-Janczyk, A., Reglinski, J., Sieklucka, B., Spicer, M. D., and Szaleniec, M.Dalton Trans. 2003, 1181

14. Senda, S., Ohki, Y., Hirayama, T., Toda, D., Chen, J.-L., Matsumoto, T., Kawaguchi, H., and Tatsumi, K.Inorg. Chem. 2006, 45, 9914

15. Figueroa, J. S., Melnick, J. G., and Parkin, G. Inorg. Chem. 2006, 45, 7056

16. (a) Silva, R. M., Gwengo, C., Smith, M. D., Lindeman, S. V., and Gardinier, J. R. Inorg. Chem. 2006, 45,10998

(b) Gardinier, J. R., Silva, R. M., Gwengo, C., andLindeman, S. V. Chem. Commun. 2007, 1524 
17. (a) Bailey, P. J., Lanfranchi, M, Marchiò, L., and Parsons, S. Inorg. Chem. 2001, 40, 5030

(b) Cammi, R., Gennari, M., Giannetto, M., Lanfranchi, M., Marchiò, L., Mori, G.,Paiola, C., and Pellinghelli, M. A. Inorg. Chem. 2005, 44, 4333

18. (a) Pearson, R. G. J. Am. Chem. Soc. 1963, 85, 3533 (b)R. G. Pearson, Ed., Hard and Soft Acids and Bases; Dowden, Hutchinson \& Ross: Stroudsburg, PA, 1973.

19. (a) Tannai, H., Tsuge, K., Sasaki, Y., Hatozaki, O., and Oyama, N. Dalton Trans. 2003, 2353

(b) Tatsuma, T., Matsui, H., Shouji, E., and Oyama, N. J. Phys. Chem. 1996, 100,14016

(c) Oyama, N., Tatsuma, T., Sato, T., and Sotomura, T. Nature (London) 1995, 373, 598

(d) Baron, M. and Wilson, C. V. J. Org. Chem. 1958,23, 1021

20. Hipler, F., Girol, S. G., Azzam, W., Fischer, R. A., and Wöll, C. Langmuir 2003, 19, 6072

21. SMART Version 5.625, SAINT+ Version 6.22 and SADABS Version 2.05; Bruker Analytical X-ray Systems, Inc.: Madison, Wisconsin, USA, 2001.

22. Sheldrick, G. M. SHELXTL Version 6.1; Bruker Analytical X-ray Systems, Inc.: Madison, Wisconsin, USA,2000.

23. Reger, D. L., Gardinier, J. R., Elgin, J. D., Smith, M. D., Hautot, D., Long, G. J., and Grandjean, F. Inorg.

Chem. 2006, 45, 8862

24. Hagen, K. S. Inorg. Chem. 2000, 39, 5864

$\underline{25}$.

Muller, A., Bogge, H., Schimanski, U., Penk, M., Nieradzik, K., Dartmann, M., Krickemeyer, E.,Schimanski, J., R omer, C., Romer, M., Dornfield, H., Wienboker, U., Hellmann, W., and Zimmermann, M.Monatsch.

Chem. 1989, 120, 367

26. Fackler, J. P., Moyer, T., Costamagna, J. A., Latorre, R., and Granifo, J. Inorg. Chem. 1987, 26, 836

27. (a) Ge, P., Haggerty, B. S., Rheingold, A. L., and Riordan, C. G. J. Am. Chem. Soc. 1994, 116, 8406

(b) Ohrenberg, C., Ge, P., Schebler, P., Riordan, C. G., Yap, G. A. P., andRheingold, A. L. Inorg.

Chem. 1996, 35, 749

28. Weighardt, K., Kuppers, H.-J., and Weiss, J. Inorg. Chem. 1985, 24, 3067

29. (a) Reger, D. L., Elgin, J. D., Smith, M. D., Grandjean, F., Rebbouh, L., and Long, G. J. Polyhedron 2006,25, 2616

(b) Reger, D. L., Gardinier, J. R., Bakbak, S., Gemmill, W.,Smith, M. D., Rebbouh, L., Grandjean, F., Shahin, A.

M., and Long, G. J. J. Am. Chem. Soc. 2005, 127,2303 (c) Reger, D. L., Gardinier, J. R., Smith, M. D., Shahin, A.

M.,Long, G. J., Rebbouh, L., and Grandjean, F. Inorg. Chem. 2005, 44, 1852

30. Shenoy, G. K., Wagner, F. E., and Kalvius, G. M. In Mössbauer Isomer Shifts; Shenoy, G. K. andWagner, F. E., Eds.; North-Holland: Amsterdam, 1978, p 49.

31. Jiao, J., Long, G. J., Rebbouh, L., Grandjean, F., Beatty, A. M., and FehIner, T. P. J. Am. Chem. Soc. 2005,127, 17819

32. Grandjean, F., Long, G. J., Hutchinson, B. B., Ohlhausen, L., Neill, P., and Holcomb, J. D. Inorg.

Chem.1989, 28, 4406

33. Rusanov, V., Stankov, S., Gushterov, V., Tsankov, L., and Trautwein, A. X. Hyperfine Interact. 2006, 169,1279

34. Winkler, H., Chumakov, A. I., and Trautwein, A. X. Top. Curr. Chem. 2004, 235, 137

35. Ingalls, R. Phys. Rev. 1964, 133, A787 